\title{
Analysis of a piecewise linear aeroelastic system with and without tuned vibration absorber
}

\author{
János Lelkes $(\mathbb{D}$ · Tamás Kalmár-Nagy
}

Received: 25 January 2020 / Accepted: 25 May 2020 / Published online: 8 September 2020

(C) The Author(s) 2020

\begin{abstract}
The dynamics of a two-degrees-of-freedom (pitch-plunge) aeroelastic system is investigated. The aerodynamic force is modeled as a piecewise linear function of the effective angle of attack. Conditions for admissible (existing) and virtual equilibria are determined. The stability and bifurcations of equilibria are analyzed. We find saddle-node, border collision and rapid bifurcations. The analysis shows that the pitchplunge model with a simple piecewise linear approximation of the aerodynamic force can reproduce the transition from divergence to the complex aeroelastic phenomenon of stall flutter. A linear tuned vibration absorber is applied to increase stall flutter wind speed and eliminate limit cycle oscillations. The effect of the absorber parameters on the stability of equilibria is investigated using the Liénard-Chipart criterion. We find that with the vibration absorber the onset of the rapid bifurcation can be shifted to higher wind speed or the oscillations can be eliminated altogether.
\end{abstract}

Keywords Piecewise linear system - Aeroelasticity . Bifurcation · Limit cycle oscillations · Linear vibration absorber

J. Lelkes $(\varangle) \cdot$ T. Kalmár-Nagy

Department of Fluid Mechanics, Faculty of Mechanical

Engineering, Budapest University of Technology and

Economics, Budapest, Hungary

e-mail: lelkes@ara.bme.hu

T. Kalmár-Nagy

e-mail: nody@kalmarnagy.com

\section{Introduction}

Nonlinear aeroelastic phenomena affect several types of aeroelastic systems such as flexible wings, helicopter rotor blades and wind turbines. Nonlinear aeroelasticity studies the interactions between inertial, elastic and aerodynamic forces on flexible structures that are exposed to airflow and feature non-negligible nonlinearity [1]. The theory of aeroelasticity is extensively covered in the literature [2-4].

The sources of nonlinearity in aeroelastic systems include geometric nonlinearity, structural nonlinearity, flow separation, friction, free-play in actuators, backlash in gears, nonlinear control laws, oscillating shock waves and other nonlinear phenomena [5,6]. A comprehensive study for such nonlinearities was presented by Lee et al. in [7] together with the derivation of the equations of motion of a 2D airfoil oscillating in pitch and plunge. Dowell et al. [8] summarized the physical basis and the effect of nonlinear aeroelasticity on the flight and its association with limit cycle oscillations (LCO). The effect of structural nonlinearities on the dynamical behavior of the system was investigated in $[9,10]$. Aerodynamic nonlinearities were studied by Dowell et al. in [11] using the describing function method. A combination of structural nonlinearity and the nonlinear ONERA stall aerodynamic model was investigated in the aeroelastic response of a nonrotating helicopter blade in the work of Tang et al. [12]. The airfoil model consisted of a NACA 0012 profile (the same used in this paper) with three types of nonlinearities: nonlin- 
ear structure linear aerodynamics, linear structure nonlinear aerodynamics and nonlinear structure with nonlinear aerodynamics. CFD-based aeroelastic investigations are described in [13-15].

Gilliatt et al. [16] studied structural and aerodynamic nonlinearities arising from stall conditions. Experimental investigation of a NACA0012 airfoil undergoing stall flutter oscillations in a low-speed wind tunnel was presented by Dimitriadis et al. in [17]. Santos et al. [18] carried out an experimental and numerical study of a NACA 0012 airfoil under the influence of structural and aerodynamic nonlinearities due to dynamic stall effects at high angles of attack. Jian et al. [19] developed a first-order, state-space model by combining a geometrically exact, nonlinear beam model with nonlinear ONERA-EDLIN dynamic stall model to investigate high-aspect-ratio flexible wings.

Nonlinear aeroelastic forces can produce complex bifurcation scenarios leading to chaotic oscillations. Sarkar et al. [20] observed a period-doubling route to chaos in the case of a 2-DOF aeroelastic model using the ONERA nonlinear aerodynamic dynamic stall model. In a recent study, Bose et al. [21] confirmed the Ruelle-Takens-Newhouse quasi-periodic route to chaos in a nonlinear aeroelastic system.

Flexible aeroelastic systems can lose stability by flutter or divergence depending on the system parameters. When flexible airfoils experience wind excitation, dynamic stability loss (called flutter instability), triggered by a Hopf bifurcation, may occur. The flow velocity for which the instability starts is called flutter velocity.

The starting point of the paper by Kalmár-Nagy et al. [22] was the observation that the coefficient of lift function can be well approximated by a piecewise linear function. Piecewise linear functions are often used to model free-play, nonlinear stiffness, nonlinear aerodynamic forces, and hysteresis nonlinearity in aeroelastic systems [10,23-25]. Free-play nonlinearity is often studied in aeroelasticity, as it is a common occurrence within the actuated control surface [26]. Sales et al. [27] studied an aeroviscoelastic system with a nonsmooth, free-play-type nonlinearities in their control surface. The dynamic aeroelastic response of multi-segmented hinged wings with bilinear stiffness characteristics was studied theoretically and experimentally in [28]. Dimitriadis et al. [29] investigated a complex mathematical wing model in unsteady flow with a piecewise linear nonlinearity.
Piecewise nonlinear functions are also used to analyze aeroelastic systems with complex piecewise nonlinear structural stiffness [30,31]. Sun et al. [32] approximated the static lift coefficient by a fourth-order piecewise polynomial function. A piecewise aerodynamic flutter equation was established by Goodman in [33], which uses a piecewise aerodynamic interpolation function. Replacing the nonlinearities of a dynamical system with piecewise linear makes the problem more analytically tractable. The phase space of piecewise linear systems consists of regions, each of which has linear dynamic equations of motion [34]. Magri et al. [35] proved that a typical airfoil section applying nonsmooth definition of the dynamic stall model can generate a nonsmooth Hopf bifurcation, similar to a rapid bifurcation.

Once the dynamics of the system is understood (at least on a rudimentary level), engineering questions can be addressed. Important questions are how to reduce the oscillation amplitude for a given range of parameters or how to control the flutter instability in an aeroelastic system. The linear tuned vibration absorber (LTVA), developed by Frahm [36], is a classical passive device for controlling flutter instabilities. This device consists of a small lumped mass attached to the primary structure through a linear spring and a damper. The first detailed study of properties and optimization of LTVAs was presented by Den Hartog [37]. If its parameters are correctly tuned, the LTVA can significantly shift the flutter speed [38]. The parameter optimization of nonlinear tuned vibration absorber for flutter control was carried out by Mahler et al. in [39]. Kassem et al. [40] presented an analytical and experimental study of an active dynamic vibration absorber for flutter control.

This work investigates the dynamics of a 2-DOF piecewise linear aeroelastic system for a broad range of the angle of attack. In Sect. 2, dimensional governing equations of the 2-DOF aeroelastic system are presented. The piecewise linear modeling of quasi-steady aerodynamic forces is summarized in Sect. 3. The piecewise linear models of lift coefficient as the function of the effective angle of attack were determined for NACA 0009, 0012, 23012 profiles. Section 4 is devoted to the nondimensionalization of the governing equations. In Sect. 5, the equilibrium points of the aeroelastic system are determined. The stability of the equilibria is investigated in Sect. 6. In Sect. 7, the classical and discontinuity-induced equilibrium bifurcations are studied. In the case of NACA 0012 and 23012 stall 


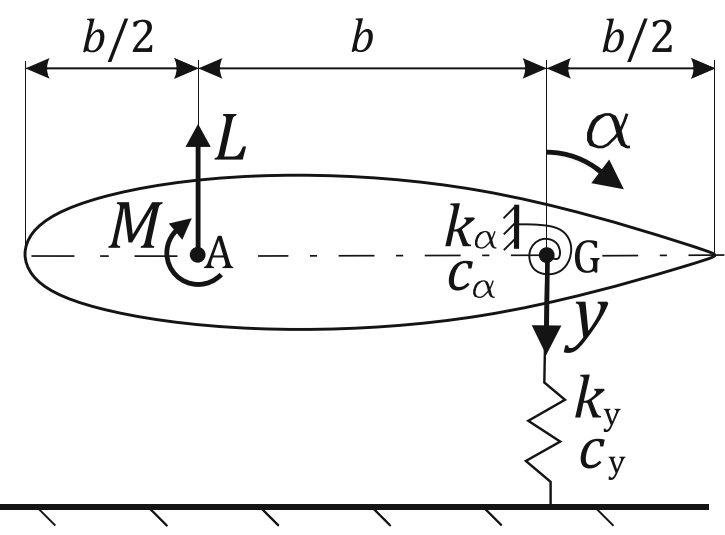

Fig. 1 The 2-DOF pitch-plunge aeroelastic model

flutter oscillations occurred. A linear tuned vibration absorber was attached to the primary aeroelastic system to eliminate these oscillations. The effect of the absorber parameters is studied in Sect. 8. The parameter optimization of the vibration absorber is investigated, showing in particular how the stall flutter can be eliminated using a well-tuned absorber. The summary and conclusions are presented in Sect. 9.

\section{The aeroelastic model}

We analyze the two-degrees-of-freedom (2-DOF) aeroelastic system shown in Fig. 1. Coordinates $y$ and $\alpha$ describe the vertical (plunge) displacement (positive downward) and angular (pitch) displacement (positive in the clockwise direction), respectively. The semichord of the airfoil is denoted by $b$. In this model, we assume that the center of gravity (denoted by $\mathbf{G}$ ) is located at three quarters of the chord length. The elastic axis passes through the center of gravity. The mass of the wing is $m$, and $I_{\mathrm{cg}}$ is the moment of inertia about the center of gravity. The linear spring constant is $k_{y}$, and the damping is $c_{y}$ for the plunge DOF. The linear spring constant is $k_{\alpha}$, and the damping is $c_{\alpha}$ for the pitch DOF. The aerodynamic lift force (positive upward) and moment (positive in the clockwise direction) applied at the airfoils aerodynamic center (denoted by $\mathbf{A}$ ) are $L$ and $M$.

The system equations are given by

$$
\begin{aligned}
& m \ddot{y}+c_{y} \dot{y}+k_{y} y=-L\left(C_{l}\left(\alpha_{\text {eff }}\right)\right), \\
& I_{\mathrm{cg}} \ddot{\alpha}+c_{\alpha} \dot{\alpha}+k_{\alpha} \alpha=M\left(C_{l}\left(\alpha_{\mathrm{eff}}\right)\right) .
\end{aligned}
$$

The aerodynamic lift force $L$ and moment $M$ are functions of the lift coefficient $C_{l}$. The lift coefficient is
Table 1 System parameters [41]

\begin{tabular}{lll}
\hline Parameter & Description & Value $/ \mathrm{units}$ \\
\hline$b$ & Semichord of wing & $0.1064 \mathrm{~m}$ \\
$S$ & Wing span & $0.6 \mathrm{~m}$ \\
$m$ & System mass & $12 \mathrm{~kg}$ \\
$I_{\mathrm{cg}}$ & Mass moment of inertia & $0.0433 \mathrm{~kg} \mathrm{~m}{ }^{2}$ \\
$k_{y}$ & Spring constant plunge DOF & $2844.4 \mathrm{~N} / \mathrm{m}$ \\
$k_{\alpha}$ & Spring constant pitch DOF & $2.82 \mathrm{Nm} / \mathrm{rad}$ \\
$c_{y}$ & Viscous damping plunge DOF & $27.43 \mathrm{~kg} / \mathrm{s}$ \\
$c_{\alpha}$ & Viscous damping pitch DOF & $0.036 \mathrm{~kg} \mathrm{~m}{ }^{2} / \mathrm{s}$ \\
$\rho$ & Air density & $1.2 \mathrm{~kg} / \mathrm{m}^{3}$ \\
\hline
\end{tabular}

a function of the effective angle of attack $\alpha_{\text {eff }}$, which takes into account the instantaneous motion of the system and the freestream velocity $U>0$ as

$\alpha_{\mathrm{eff}}=\alpha+\frac{\dot{y}}{U}$.

The system parameters used in this study were taken from the paper by Gilliatt et al. [41] and are summarized in Table 1.

\section{Aerodynamic forces}

The aerodynamic lift force and moment on the righthand side of Eq. (1) are assumed to be proportional to the lift coefficient, i.e.,

$$
\begin{aligned}
& L\left(C_{l}\left(\alpha_{\text {eff }}\right)\right)=\rho U^{2} S b C_{l}\left(\alpha_{\text {eff }}\right), \\
& M\left(C_{l}\left(\alpha_{\text {eff }}\right)\right)=\rho U^{2} S b^{2} C_{l}\left(\alpha_{\text {eff }}\right),
\end{aligned}
$$

where $\rho$ is the air density, $S$ is the wing span, and $b$ is the semichord. The measured static lift coefficient versus effective angle of attack for different airfoil sections $[42,43]$ is illustrated in Fig. 2.

Kalmár-Nagy et al. [22] introduced a piecewise linear continuous model for the aerodynamic lift coefficient. Here we extend this model for a larger range of $\alpha_{\text {eff }}$. The piecewise linear continuous model consists of five regions $\left(-\infty, \alpha_{\text {switch }}^{-}\right],\left[\alpha_{\text {switch }}^{-}, \alpha_{\text {stall }}^{-}\right]$, $\left[\alpha_{\text {stall }}^{-}, \alpha_{\text {stall }}^{+}\right],\left[\alpha_{\text {stall }}^{+}, \alpha_{\text {switch }}^{+}\right],\left[\alpha_{\text {switch }}^{+}, \infty\right)$ as shown in Fig. 3.

The lift coefficient is given as the piecewise linear function 


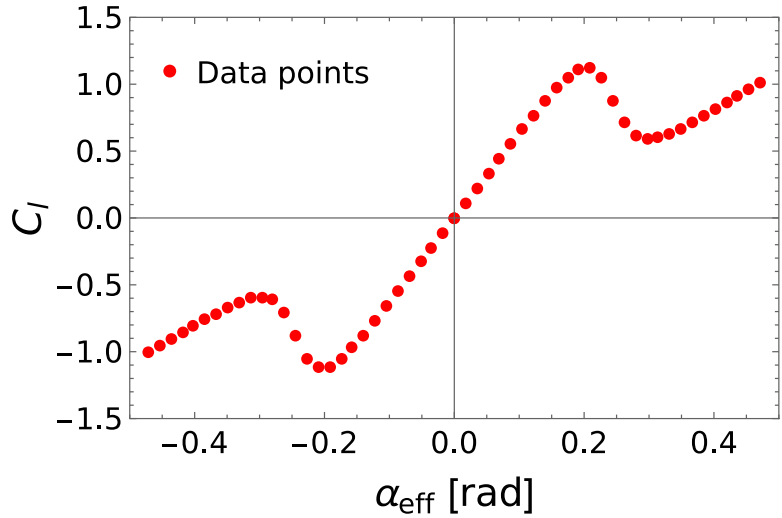

(a) NACA0012 measured data [42]

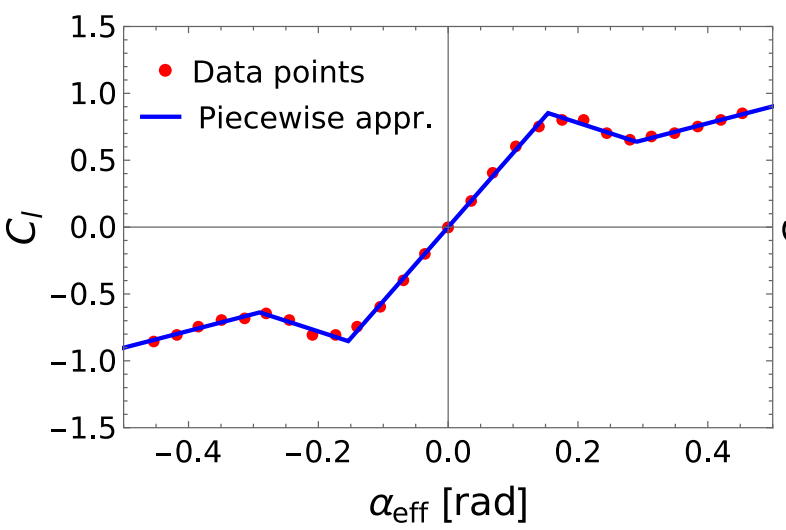

(c) NACA0009 piecewise linear fit [42]

Fig. 2 Data points and piecewise fitted models

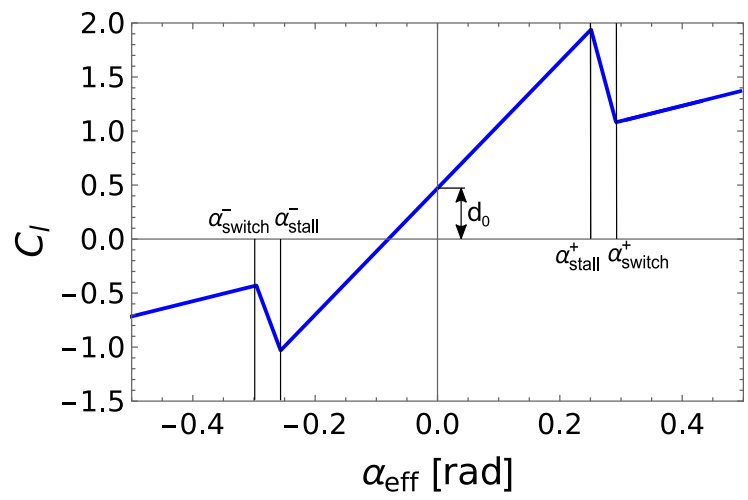

Fig. 3 Piecewise linear model of the aerodynamic lift coefficient

$C_{l}\left(\alpha_{\mathrm{eff}}\right)= \begin{cases}c_{2}^{-} \alpha_{\mathrm{eff}}+d_{2}^{-} & \alpha_{\mathrm{eff}} \in\left(-\infty, \alpha_{\mathrm{switch}}^{-}\right], \\ c_{1}^{-} \alpha_{\mathrm{eff}}+d_{1}^{-} & \alpha_{\mathrm{eff}} \in\left[\alpha_{\text {switch }}^{-}, \alpha_{\text {stall }}^{-}\right], \\ c_{0} \alpha_{\mathrm{eff}}+d_{0} & \alpha_{\mathrm{eff}} \in\left[\alpha_{\text {stall }}^{-}, \alpha_{\text {stall }}^{+}\right], \\ c_{1}^{+} \alpha_{\mathrm{eff}}+d_{1}^{+} & \alpha_{\mathrm{eff}} \in\left[\alpha_{\text {stall }}^{+}, \alpha_{\mathrm{switch}}^{+}\right], \\ c_{2}^{+} \alpha_{\mathrm{eff}}+d_{2}^{+} & \alpha_{\mathrm{eff}} \in\left[\alpha_{\text {switch }}^{+}, \infty\right) .\end{cases}$

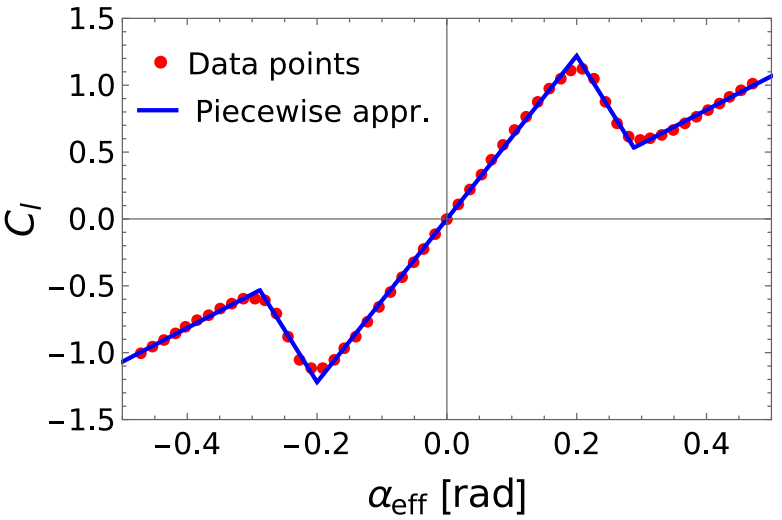

(b) NACA0012 piecewise linear fit

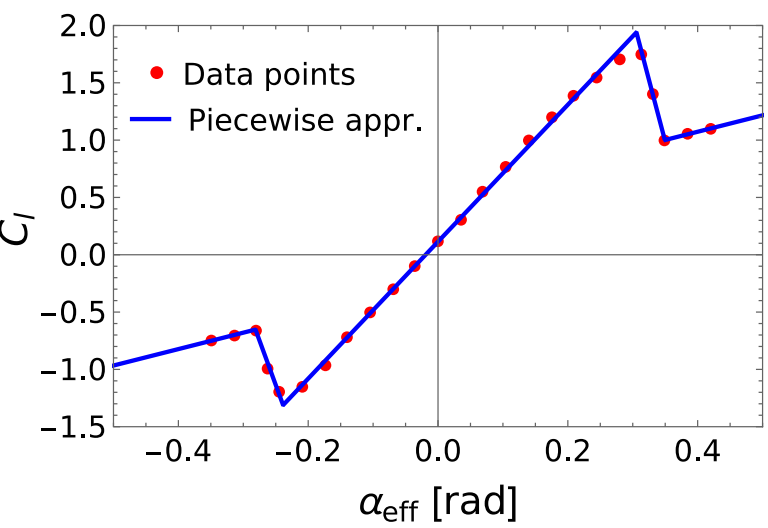

(d) NACA 23012 piecewise linear fit [43]

Parameter $\alpha_{\text {stall }}^{+}$characterizes the stall condition for positive $\alpha_{\text {eff }}$ at which lift starts to decrease as $\alpha_{\text {eff }}$ is increased. Parameter $\alpha_{\text {switch }}^{+}$corresponds to the switching point at which the slope of $C_{l}$ starts to increase again. The negative angles of attack $\alpha_{\text {stall }}^{-}$and $\alpha_{\text {switch }}^{-}$ are defined analogously. We also impose the following continuity constraints to hold

$$
\begin{aligned}
& c_{2}^{-} \alpha_{\text {switch }}^{-}+d_{2}^{-}=c_{1}^{-} \alpha_{\text {switch }}^{-}+d_{1}^{-}, \\
& c_{1}^{-} \alpha_{\text {stall }}^{-}+d_{1}^{-}=c_{0} \alpha_{\text {stall }}^{-}+d_{0}, \\
& c_{0} \alpha_{\text {stall }}^{+}+d_{0}=c_{1}^{+} \alpha_{\text {stall }}^{+}+d_{1}^{+}, \\
& c_{1}^{+} \alpha_{\text {switch }}^{+}+d_{1}^{+}=c_{2}^{+} \alpha_{\text {switch }}^{+}+d_{2}^{+} .
\end{aligned}
$$

Symmetric profiles give rise to an odd coefficient of lift function with parameters

$$
\begin{gathered}
\alpha_{\text {stall }}^{+}=-\alpha_{\text {stall }}^{-}=\alpha_{\text {stall }}, \\
\alpha_{\text {switch }}^{+}=-\alpha_{\text {switch }}^{-}=\alpha_{\text {switch }}, \\
d_{0}=0, \\
c_{1}^{+}=c_{1}^{-}=c_{1}, \quad d_{1}^{+}=-d_{1}^{-}=d_{1}, \\
c_{2}^{+}=c_{2}^{-}=c_{2}, \quad d_{2}^{+}=-d_{2}^{-}=d_{2} .
\end{gathered}
$$


Table 2 Piecewise linear fit parameters

\begin{tabular}{llll}
\hline Parameter & NACA 0012 & NACA 0009 & NACA 23012 \\
\hline$\alpha_{\text {switch }}^{-}$ & -0.296 & -0.290 & -0.281 \\
$\alpha_{\text {stall }}^{-}$ & -0.201 & -0.154 & -0.239 \\
$\alpha_{\text {stall }}^{+}$ & 0.201 & 0.154 & 0.306 \\
$\alpha_{\text {switch }}^{+}$ & 0.296 & 0.290 & 0.349 \\
$c_{2}^{-}$ & 2.662 & 1.261 & 1.432 \\
$d_{2}^{-}$ & 0.256 & -0.272 & -0.250 \\
$c_{1}^{-}$ & -6.846 & -1.576 & -15.47 \\
$d_{1}^{-}$ & -2.556 & -1.095 & -5.033 \\
$c_{0}$ & 5.932 & 5.539 & 5.973 \\
$d_{0}$ & 0 & 0 & 0.114 \\
$c_{1}^{+}$ & -6.846 & -1.576 & -21.49 \\
$d_{1}^{+}$ & 2.556 & 1.095 & 8.508 \\
$c_{2}^{+}$ & 2.662 & 1.261 & 1.432 \\
$d_{2}^{+}$ & -0.256 & 0.272 & 0.501 \\
\hline
\end{tabular}

The piecewise linear models of the lift coefficient for the NACA 0012, 0009 and 23012 profiles are shown in Fig. 2. The parameters $c_{0}, d_{0}, c_{1}^{ \pm}, d_{1}^{ \pm}, c_{2}^{ \pm}, d_{2}^{ \pm}$were determined by least square method using piecewise linear model (4) and continuity constraints (5). The parameters are given in Table 2 . We point out that the NACA 0012, 0009 profiles are symmetric, while NACA 23012 is asymmetric.

In the next section, the governing nondimensional equations are derived for symmetric airfoil profiles (for the nonsymmetric case the derivation can be done similarly).

\section{Nondimensional equations for symmetric profiles}

Nondimensionalization results in a better understanding of the physical phenomenon (via the nondimensional groups obtained) and in a reduction of system parameters [44]. Following [22] we choose the length scale $\mathcal{L}$, the timescale $\mathcal{T}$, and the nondimensional freestream velocity $\mu>0$ as

$$
\begin{aligned}
& \mathcal{L}=\sqrt{\frac{I_{\mathrm{cg}}}{\rho b^{2} S}}, \quad \mathcal{T}=\sqrt{\frac{m}{k_{y}}}, \\
& \mu=\frac{U}{\mathcal{L} / \mathcal{T}}=\sqrt{\frac{m \rho b^{2} S}{k_{y} I_{\mathrm{cg}}}} U .
\end{aligned}
$$

These scales yield the nondimensional plunge $\hat{y}=$ $y / \mathcal{L}$, the nondimensional time $\tau=t / \mathcal{T}$. The derivative with respect to the nondimensional time is denoted by ()$^{\prime}=\mathrm{d}() / \mathrm{d} \tau$. The nondimensional effective angle of attack can now be expressed as

$\alpha_{\text {eff }}=\alpha+\frac{1}{\mu} \hat{y}^{\prime}$.

The nondimensional form of Eq. (1) is

$$
\begin{aligned}
& \hat{y}^{\prime \prime}+p_{1} \hat{y}^{\prime}+\hat{y}=-p_{2} \mu^{2} C_{l}\left(\alpha+\frac{1}{\mu} \hat{y}^{\prime}\right), \\
& \alpha^{\prime \prime}+p_{3} \alpha^{\prime}+p_{4} \alpha=\mu^{2} C_{l}\left(\alpha+\frac{1}{\mu} \hat{y}^{\prime}\right),
\end{aligned}
$$

where the nondimensional parameters $p_{1}, p_{2}, p_{3}$ and $p_{4}$ are

$$
\begin{aligned}
& p_{1}=\frac{c_{y}}{\sqrt{m k_{y}}}, \quad p_{2}=\frac{\sqrt{\rho I_{\mathrm{cg}} S}}{m}, \\
& p_{3}=\frac{c_{\alpha}}{I_{\mathrm{cg}}} \sqrt{\frac{m}{k_{y}}}, \quad p_{4}=\frac{k_{\alpha} m}{I_{\mathrm{cg}} k_{y}} .
\end{aligned}
$$

Using trilinear approximation (4) of the lift coefficient for symmetric profiles (see Eq. (6)), we obtain the following equations:

for $\left|\alpha_{\text {eff }}\right| \leq \alpha_{\text {stall }}$

$$
\begin{aligned}
& \hat{y}^{\prime \prime}+\left(p_{1}+p_{2} \mu c_{0}\right) \hat{y}^{\prime}+\hat{y}+p_{2} \mu^{2} c_{0} \alpha=0, \\
& \alpha^{\prime \prime}+p_{3} \alpha^{\prime}+\left(p_{4}-\mu^{2} c_{0}\right) \alpha-\mu c_{0} \hat{y}^{\prime}=0,
\end{aligned}
$$

for $\alpha_{\text {stall }} \leq\left|\alpha_{\text {eff }}\right| \leq \alpha_{\text {switch }}$

$$
\begin{aligned}
& \hat{y}^{\prime \prime}+\left(p_{1}+p_{2} \mu c_{1}\right) \hat{y}^{\prime}+\hat{y}+p_{2} \mu^{2} c_{1} \alpha \\
& \quad+\operatorname{sgn}\left(\alpha_{\text {eff }}\right) p_{2} \mu^{2} d_{1}=0, \\
& \alpha^{\prime \prime}+p_{3} \alpha^{\prime}+\left(p_{4}-\mu^{2} c_{1}\right) \alpha-\mu c_{1} \hat{y}^{\prime} \\
& \quad-\operatorname{sgn}\left(\alpha_{\text {eff }}\right) \mu^{2} d_{1}=0,
\end{aligned}
$$

and for $\alpha_{\text {switch }} \leq\left|\alpha_{\text {eff }}\right|$

$$
\begin{aligned}
& \hat{y}^{\prime \prime}+\left(p_{1}+p_{2} \mu c_{2}\right) \hat{y}^{\prime}+\hat{y}+p_{2} \mu^{2} c_{2} \alpha \\
& \quad+\operatorname{sgn}\left(\alpha_{\text {eff }}\right) p_{2} \mu^{2} d_{2}=0, \\
& \alpha^{\prime \prime}+p_{3} \alpha^{\prime}+\left(p_{4}-\mu^{2} c_{2}\right) \alpha-\mu c_{2} \hat{y}^{\prime} \\
& \quad-\operatorname{sgn}\left(\alpha_{\text {eff }}\right) \mu^{2} d_{2}=0 .
\end{aligned}
$$

We introduce the state vector

$\boldsymbol{x}=\left[\begin{array}{c}\hat{y} \\ \hat{y}^{\prime} \\ \alpha \\ \alpha^{\prime}\end{array}\right]$,

the system matrices

$\boldsymbol{A}_{k}=\left[\begin{array}{cccc}0 & 1 & 0 & 0 \\ -1 & -\left(p_{1}+p_{2} \mu c_{k}\right) & -p_{2} \mu^{2} c_{k} & 0 \\ 0 & 0 & 0 & 1 \\ 0 & \mu c_{k} & -\left(p_{4}-\mu^{2} c_{k}\right) & -p_{3}\end{array}\right], k=0,1,2$, 
and translation vectors

$\boldsymbol{b}_{k}=\left[\begin{array}{c}0 \\ -p_{2} \mu^{2} d_{k} \\ 0 \\ \mu^{2} d_{k}\end{array}\right], k=1,2$.

Equations (11)-(13) can now be concisely written as a collection of affine systems, together with their domains of validity

$\dot{x}=A_{2} x-b_{2}, \quad x \in \Omega_{2}^{-} \cup \Sigma_{2}^{-}$,

$\dot{x}=A_{1} x-b_{1}, \quad x \in \Sigma_{2}^{-} \cup \Omega_{1}^{-} \cup \Sigma_{1}^{-}$,

$\dot{x}=A_{0} x, \quad x \in \Sigma_{1}^{-} \cup \Omega_{0} \cup \Sigma_{1}^{+}$,

$\dot{\boldsymbol{x}}=\boldsymbol{A}_{1} \boldsymbol{x}+\boldsymbol{b}_{1}, \quad \boldsymbol{x} \in \Sigma_{1}^{+} \cup \Omega_{1}^{+} \cup \Sigma_{2}^{+}$,

$\dot{x}=A_{2} x+b_{2}, \quad x \in \Sigma_{2}^{+} \cup \Omega_{2}^{+}$,

where the domains of subsystems (17)-(21) are given by

$\Omega_{2}^{-}:=\left\{x \in \mathbb{R}^{4}: \alpha+\frac{\hat{y}^{\prime}}{\mu} \in\left(-\infty,-\alpha_{\text {switch }}\right)\right\}$,

$\Omega_{1}^{-}:=\left\{x \in \mathbb{R}^{4}: \alpha+\frac{\hat{y}^{\prime}}{\mu} \in\left(-\alpha_{\text {switch }},-\alpha_{\text {stall }}\right)\right\}$

$\Omega_{0}:=\left\{x \in \mathbb{R}^{4}: \alpha+\frac{\hat{y}^{\prime}}{\mu} \in\left(-\alpha_{\text {stall }}, \alpha_{\text {stall }}\right)\right\}$,

$\Omega_{1}^{+}:=\left\{\boldsymbol{x} \in \mathbb{R}^{4}: \alpha+\frac{\hat{y}^{\prime}}{\mu} \in\left(\alpha_{\text {stall }}, \alpha_{\text {switch }}\right)\right\}$,

$\Omega_{2}^{+}:=\left\{x \in \mathbb{R}^{4}: \alpha+\frac{\hat{y}^{\prime}}{\mu} \in\left(\alpha_{\text {switch }}, \infty\right)\right\}$.

These domains are separated by the switching planes defined by

$\Sigma_{1}^{ \pm}:=\left\{x \in \mathbb{R}^{4}: \alpha+\frac{\hat{y}^{\prime}}{\mu}= \pm \alpha_{\text {stall }}\right\}$,

$\Sigma_{2}^{ \pm}:=\left\{x \in \mathbb{R}^{4}: \alpha+\frac{\hat{y}^{\prime}}{\mu}= \pm \alpha_{\text {switch }}\right\}$.

With these pairwise disjoint sets, the full state space $\mathbb{R}^{4}$ can be decomposed as

$$
\begin{aligned}
\mathbb{R}^{4}= & \Omega_{2}^{-} \cup \Sigma_{2}^{-} \cup \Omega_{1}^{-} \cup \Sigma_{1}^{-} \\
& \cup \Omega_{0} \cup \Sigma_{1}^{+} \cup \Omega_{1}^{+} \cup \Sigma_{2}^{+} \cup \Omega_{2}^{+} .
\end{aligned}
$$

\section{Equilibrium points}

The equilibrium points of system (17)-(21) are formally given by $\dot{\boldsymbol{x}}=\mathbf{0}$. Clearly, an equilibrium point only exists if it is inside the corresponding domain of validity. Following Di Bernardo et al. [45] we refer to such an equilibrium point as admissible. We call an equilibrium point virtual if it is not admissible (i.e., it satisfies $\dot{\boldsymbol{x}}=\mathbf{0}$ but falls outside the domain of validity). Virtual equilibria play an important role in organizing the dynamics of the corresponding region [46]. The admissible equilibrium points of system (17)-(21) are

$$
\begin{aligned}
\boldsymbol{E}_{0}=\mathbf{0}, \quad \boldsymbol{E}_{0} \in \Sigma_{1}^{-} \cup \Omega_{0} \cup \Sigma_{1}^{+}, \\
\boldsymbol{E}_{1}^{ \pm}=\mp \boldsymbol{A}_{1}^{-1} \boldsymbol{b}_{1}=\mp \frac{d_{1} \mu^{2}}{c_{1} \mu^{2}-p_{4}}\left[\begin{array}{c}
-p_{2} p_{4} \\
0 \\
1 \\
0
\end{array}\right], \\
\boldsymbol{E}_{1}^{ \pm} \in \Sigma_{1}^{ \pm} \cup \Omega_{1}^{ \pm} \cup \Sigma_{2}^{ \pm}, \\
\boldsymbol{E}_{2}^{ \pm}=\mp \boldsymbol{A}_{2}^{-1} \boldsymbol{b}_{2}=\mp \frac{d_{2} \mu^{2}}{c_{2} \mu^{2}-p_{4}}\left[\begin{array}{c}
-p_{2} p_{4} \\
0 \\
1 \\
0
\end{array}\right], \\
\boldsymbol{E}_{2}^{ \pm} \in \Sigma_{2}^{ \pm} \cup \Omega_{2}^{ \pm} .
\end{aligned}
$$

We express the admissibility conditions embedded in Eqs. (30)-(32) in terms of the system parameters.

$\boldsymbol{E}_{\mathbf{0}}$ is always admissible for symmetric airfoil profiles,

$$
\begin{aligned}
& \boldsymbol{E}_{1}^{+} \text {is admissible if } \alpha_{\text {stall }} \\
& \leq-\frac{d_{1} \mu^{2}}{c_{1} \mu^{2}-p_{4}} \leq \alpha_{\text {switch }},
\end{aligned}
$$

$\boldsymbol{E}_{2}^{+}$is admissible if $\alpha_{\text {switch }}$

$$
\leq-\frac{d_{2} \mu^{2}}{c_{2} \mu^{2}-p_{4}} \text {. }
$$

From admissibility condition (34) we obtain that equilibrium $\boldsymbol{E}_{1}^{+}$is admissible if $\mu \in\left[\mu_{1}, \mu_{2}\right]$, where

$$
\begin{aligned}
& \mu_{1}=\sqrt{\frac{p_{4} \alpha_{\text {stall }}}{d_{1}+c_{1} \alpha_{\text {stall }}}}, \\
& \mu_{2}=\sqrt{\frac{p_{4} \alpha_{\text {switch }}}{d_{1}+c_{1} \alpha_{\text {switch }}}} .
\end{aligned}
$$

One can utilize continuity condition (5) and symmetry condition (6) to express $\mu_{1}$ as

$\mu_{1}=\sqrt{\frac{p_{4}}{c_{0}}}$.

Let us define (provided $c_{2}>0$ )

$\mu_{A}=\sqrt{\frac{p_{4}}{c_{2}}}$.

From condition (35) we find that equilibrium $\boldsymbol{E}_{2}^{+}$is admissible in $\mu \in\left(\mu_{A}, \mu_{2}\right]\left(\mu \in\left[\mu_{2}, \mu_{A}\right)\right)$ if $\mu_{A}<$ $\mu_{2}\left(\mu_{A}>\mu_{2}\right)$. 


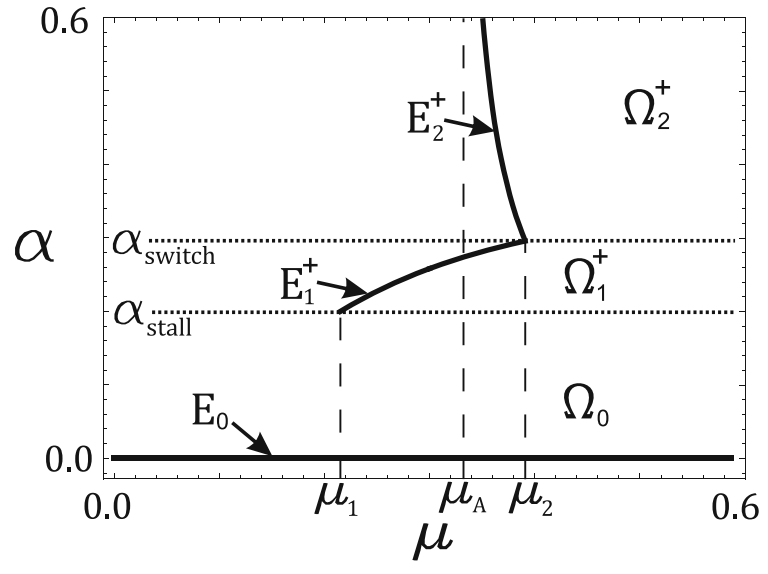

(a) NACA 0012

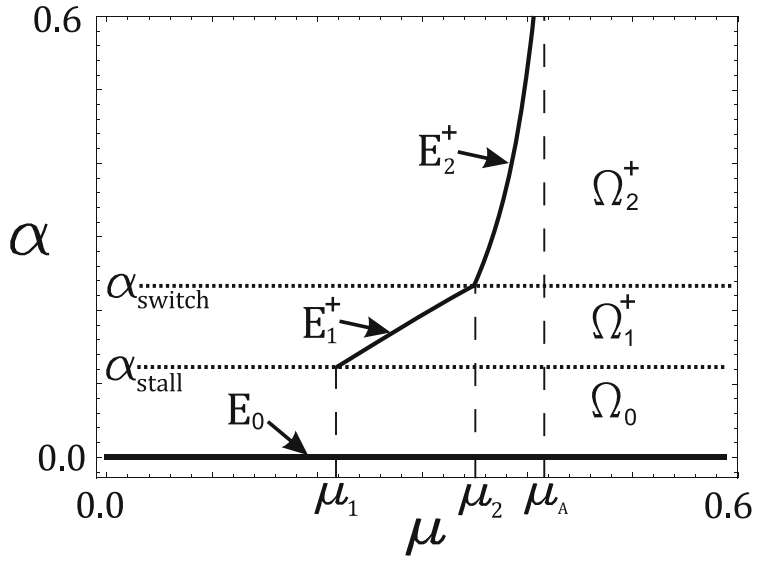

(b) NACA 0009

Fig. 4 The admissible equilibria of the symmetric aeroelastic models

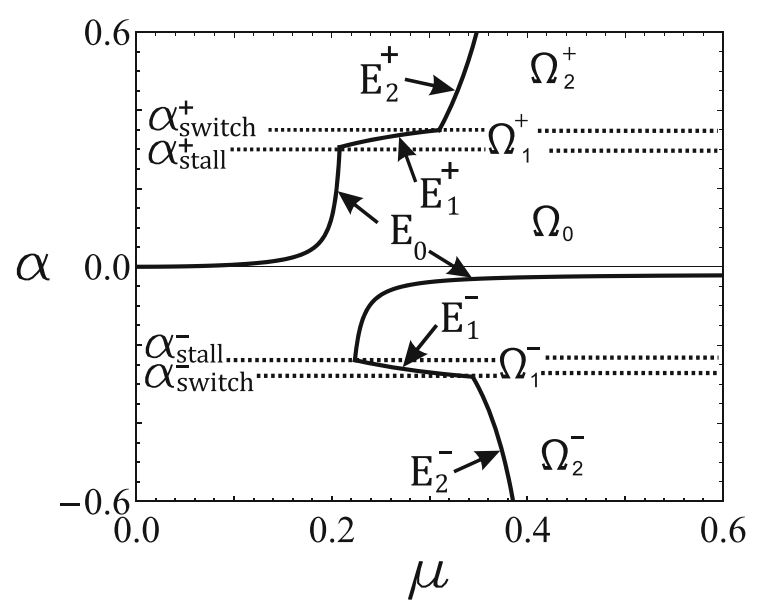

Fig. 5 The admissible equilibria of the asymmetric aeroelastic model NACA 23012

Systems (17)-(21) is symmetric; therefore, $\boldsymbol{E}_{1}^{-}$and $\boldsymbol{E}_{2}^{-}$have the same admissibility range of $\mu$ as $\boldsymbol{E}_{1}^{+}$and $\boldsymbol{E}_{2}^{+}$.

The admissible equilibria of system (17)-(21) in the $(\mu, \alpha)$ plane are illustrated in Fig. 4 for the parameters of the NACA 0012 and NACA 0009 profiles (listed in Tables 1 and 2). Even though the derivation here does not include formulae for asymmetric profiles, we nevertheless show admissible equilibria for the aerodynamic parameters of the asymmetric NACA 23012 profile in Fig. 5. In Figs. 4 and 5 the solid lines correspond to the admissible equilibrium points, and the dotted lines are the switching lines defined in Eqs. (27) and (28).

\section{Classical stability of equilibria}

In this section the asymptotic stability of equilibrium points (30)-(32) is investigated (these correspond to symmetric profiles). We apply the Liénard-Chipart criterion [47] to the characteristic polynomials $R_{k}(\lambda, \mu)$ of the coefficient matrices $\boldsymbol{A}_{k}$ given in Eq. (15) to determine the necessary and sufficient conditions for asymptotic stability. The stability criterion of Liénard and Chipart expresses the necessary and sufficient conditions for the stability of polynomials in terms of the coefficients and the so-called Hurwitz determinants.

Theorem (Stability Criterion of Liénard and Chipart [47]): Necessary and sufficient conditions for all the roots of the real $n$ th-degree polynomial $R(\lambda)=\lambda^{n}+$ $a_{1} \lambda^{n-1}+\cdots+a_{n-1} \lambda+a_{n}$, to have negative real parts, can be given in any one of the following forms:

1) $a_{n}>0, a_{n-2}>0, \ldots ; \Delta_{1}>0, \Delta_{3}>0, \ldots$

2) $a_{n}>0, a_{n-2}>0, \ldots ; \Delta_{2}>0, \Delta_{4}>0, \ldots$

3) $a_{n}>0 ; a_{n-1}>0, a_{n-3}>0, \ldots ; \Delta_{1}>0, \Delta_{3}>0, \ldots$

4) $a_{n}>0 ; a_{n-1}>0, a_{n-3}>0, \ldots ; \Delta_{2}>0, \Delta_{4}>0, \ldots$

Here

$$
\Delta_{i}=\left|\begin{array}{cccccc}
a_{1} & a_{3} & a_{5} & \ldots & & \\
1 & a_{2} & a_{4} & \ldots & & \\
0 & a_{1} & a_{3} & \ldots & & \\
0 & 1 & a_{2} & a_{4} & & \\
& & & & \ddots & \\
& & & & & a_{i}
\end{array}\right|, \quad\left(a_{k}=0 \text { for } k>n\right)
$$




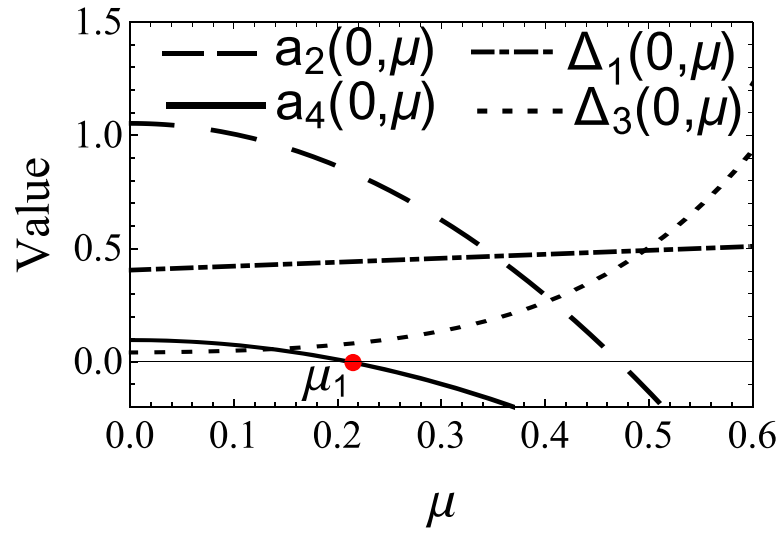

(a) $k=0$

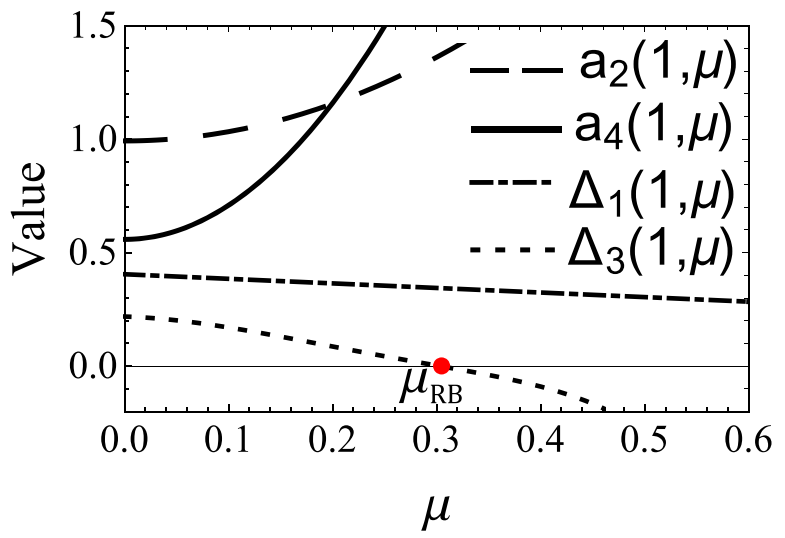

(b) $k=1$

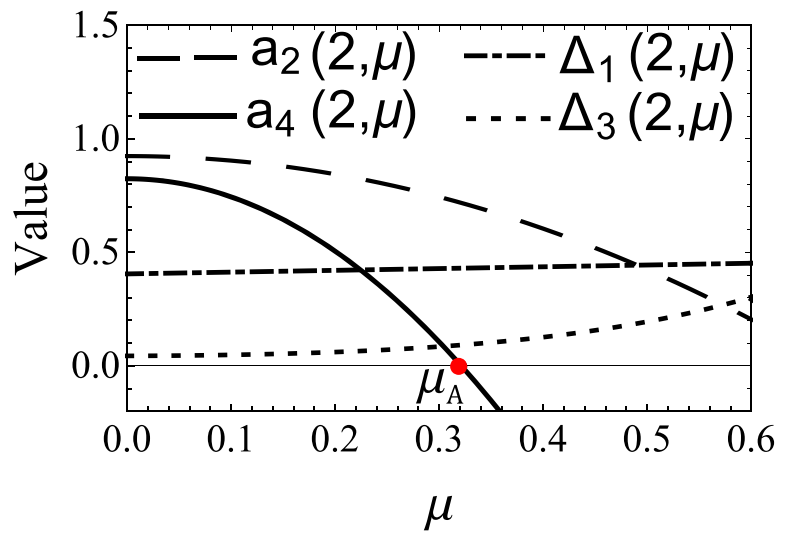

(c) $k=2$

Fig. 6 The expressions $a_{2}(k, \mu), a_{4}(k, \mu), \Delta_{1}(k, \mu)$ and $\Delta_{3}(k, \mu)$ as the function of $\mu$

are the Hurwitz determinant of order $i$, e.g.,

$$
\begin{aligned}
\Delta_{1} & =a_{1}, \\
\Delta_{3} & =\left|\begin{array}{ccc}
a_{1} & a_{3} & a_{5} \\
1 & a_{2} & a_{4} \\
0 & a_{1} & a_{3}
\end{array}\right|, \\
\Delta_{5} & =\left|\begin{array}{ccccc}
a_{1} & a_{3} & a_{5} & a_{7} & a_{9} \\
1 & a_{2} & a_{4} & a_{6} & a_{8} \\
0 & a_{1} & a_{3} & a_{5} & a_{7} \\
0 & 1 & a_{2} & a_{4} & a_{6} \\
0 & 0 & a_{1} & a_{3} & a_{5}
\end{array}\right| .
\end{aligned}
$$

In this paper we use the Liénard-Chipart criterion for 4th- and 6th-degree polynomials. The easiestto-apply Liénard-Chipart criterion for our 4th-degree polynomials $R(\lambda)=\lambda^{4}+a_{1} \lambda^{3}+a_{2} \lambda^{2}+a_{3} \lambda+a_{4}$ is

$$
\begin{aligned}
& a_{2}>0, a_{4}>0 ; \Delta_{1}=a_{1}>0, \\
& \Delta_{3}=a_{1} a_{2} a_{3}-a_{1}^{2} a_{4}-a_{3}^{2}>0,
\end{aligned}
$$

while for the 6th-degree polynomials $R(\lambda)=\lambda^{6}+$ $a_{1} \lambda^{5}+a_{2} \lambda^{4}+a_{3} \lambda^{3}+a_{4} \lambda^{2}+a_{5} \lambda+a_{6}$ of Sect. 8 is

$$
\begin{aligned}
a_{2}> & 0, a_{4}>0, a_{6}>0 \\
\Delta_{1}= & a_{1}>0, \Delta_{3}=a_{1} a_{2} a_{3}+a_{1} a_{5} \\
& -a_{1}^{2} a_{4}-a_{3}^{2}>0, \\
\Delta_{5}= & a_{1} a_{2} a_{3} a_{4} a_{5}-a_{6}^{2} a_{1}^{3}-a_{4}^{2} a_{5} a_{1}^{2} \\
& +a_{3} a_{4} a_{6} a_{1}^{2}+2 a_{2} a_{5} a_{6} a_{1}^{2}-a_{2}^{2} a_{5}^{2} a_{1} \\
& +2 a_{4} a_{5}^{2} a_{1}-a_{2} a_{3}^{2} a_{6} a_{1}-3 a_{3} a_{5} a_{6} a_{1} \\
& -a_{5}^{3}+a_{2} a_{3} a_{5}^{2}-a_{3}^{2} a_{4} a_{5}+a_{3}^{3} a_{6}>0 .
\end{aligned}
$$

The characteristic polynomials $R_{k}(\lambda, \mu)$ of the coefficient matrices $\boldsymbol{A}_{k}$ given in Eq. (15) are 


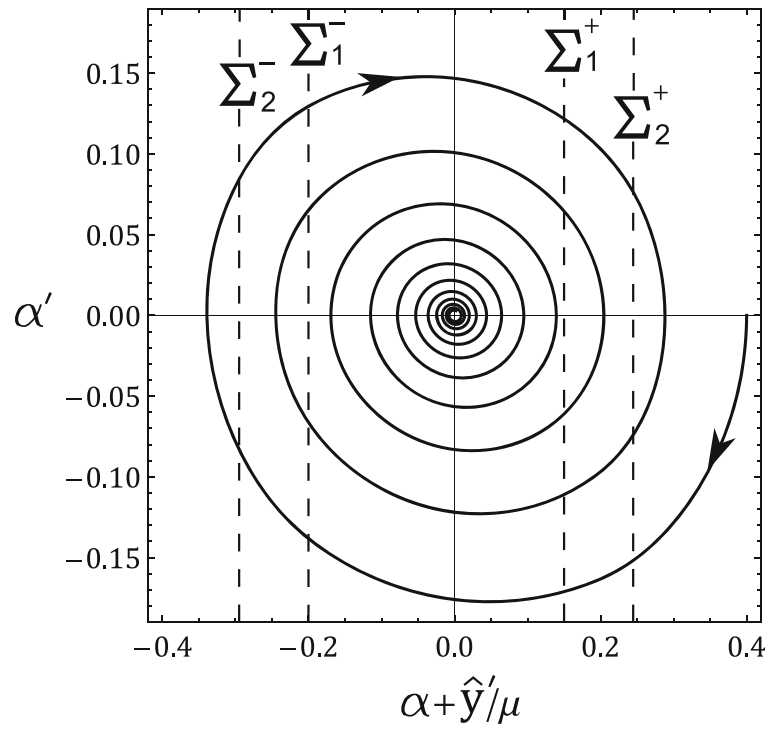

(a) $\mu=0.15<\mu_{1}=0.215$

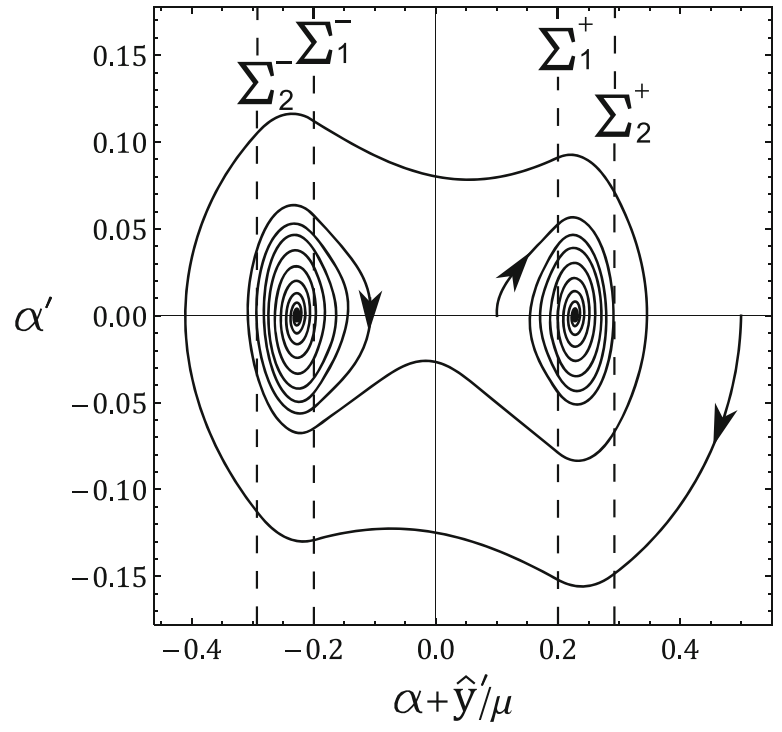

(b) $\mu=0.25>\mu_{1}=0.215$

Fig. 7 Typical trajectories $\mathbf{a}$ before and $\mathbf{b}$ after the first-border collision

$$
\begin{aligned}
R_{k}(\lambda, \mu)= & \operatorname{det}\left(\boldsymbol{A}_{k}-\lambda \boldsymbol{I}\right)=\lambda^{4}+a_{1}(k, \mu) \lambda^{3} \\
& +a_{2}(k, \mu) \lambda^{2}+a_{3}(k, \mu) \lambda+a_{4}(k, \mu),
\end{aligned}
$$

where

$a_{1}(k, \mu)=p_{3}+p_{1}+p_{2} c_{k} \mu$,

$a_{2}(k, \mu)=1+p_{4}+p_{1} p_{3}+p_{2} p_{3} c_{k} \mu-c_{k} \mu^{2}$,

$a_{3}(k, \mu)=p_{3}+p_{1} p_{4}+p_{2} p_{4} c_{k} \mu-p_{1} c_{k} \mu^{2}$,

$a_{4}(k, \mu)=p_{4}-c_{k} \mu^{2}$.

Expression $a_{1}(k, \mu)$ is a linear function of $\mu$, and $a_{2}(k, \mu), a_{3}(k, \mu) a_{4}(k, \mu)$ are quadratic polynomials of $\mu$. The Hurwitz determinants expressed by the system parameters are

$$
\begin{aligned}
\Delta_{1}(k, \mu)= & p_{3}+p_{1}+p_{2} c_{k} \mu \\
\Delta_{3}(k, \mu)= & p_{1} p_{3}\left(p_{3}^{2}+\left(p_{4}-1\right)^{2}+p_{1}^{2} p_{4}\right. \\
& \left.+p_{1} p_{3}\left(p_{4}+1\right)\right) \\
& +p_{2} p_{3} c_{k}\left(3 p_{4} p_{1}^{2}+2 p_{3}\left(p_{4}+1\right) p_{1}\right. \\
& \left.+p_{3}^{2}+\left(p_{4}-1\right)^{2}\right) \mu \\
& +p_{3} c_{k}\left(p_{1}\left(p_{4}\left(3 p_{2}^{2} c_{k}-2\right)+2\right)\right. \\
& \left.+p_{2}^{2} p_{3}\left(p_{4}+1\right) c_{k}-p_{1}^{2}\left(p_{1}+p_{3}\right)\right) \mu^{2}
\end{aligned}
$$

$$
\begin{aligned}
& +p_{2} c_{k}^{2}\left(p_{3}\left(p_{4}\left(p_{2}^{2} c_{k}-1\right)+1\right)\right. \\
& \left.-2 p_{3} p_{1}^{2}-\left(p_{3}^{2}+p_{4}-1\right) p_{1}\right) \mu^{3} \\
& +c_{k}^{2}\left(p_{1} p_{3}-p_{2}^{2}\left(p_{1} p_{3}+p_{4}-1\right) c_{k}\right) \mu^{4} \\
& +c_{k}^{3} p_{1} p_{2} \mu^{5}
\end{aligned}
$$

To determine the point of stability loss (bifurcation) of equilibria (30)-(32), we need to find the smallest positive $\mu$ for which any of inequalities (44) is violated. In other words, we are looking for the smallest positive root $\mu$ of any of

$$
\begin{aligned}
& a_{2}(k, \mu)=0, a_{4}(k, \mu)=0, \Delta_{1}(k, \mu)=0, \\
& \Delta_{3}(k, \mu)=0, \quad k=0,1,2 .
\end{aligned}
$$

Now we show the results of stability analysis for structural and aerodynamic parameters of the model with a NACA 0012 profile (Tables 1 and 2). For the equilibrium points $\boldsymbol{E}_{0}, \boldsymbol{E}_{1}^{ \pm}, \boldsymbol{E}_{2}^{ \pm}$defined in Eqs. (30)(32) condition (44) is fulfilled at $\mu=0$ (see Fig. 6). For $\boldsymbol{E}_{0}$ the Hurwitz determinants $\Delta_{1}(0, \mu), \Delta_{3}(0, \mu)$ are positive for $\mu \geq 0$, since

$$
\begin{aligned}
\Delta_{1}(0, \mu)= & 0.2025+0.0873 \mu>0, \\
\Delta_{3}(0, \mu)= & 0.0044+0.0027 \mu+0.0675 \mu^{2} \\
& +0.0746 \mu^{3}+0.3145 \mu^{4}+0.4560 \mu^{5}>0 .
\end{aligned}
$$




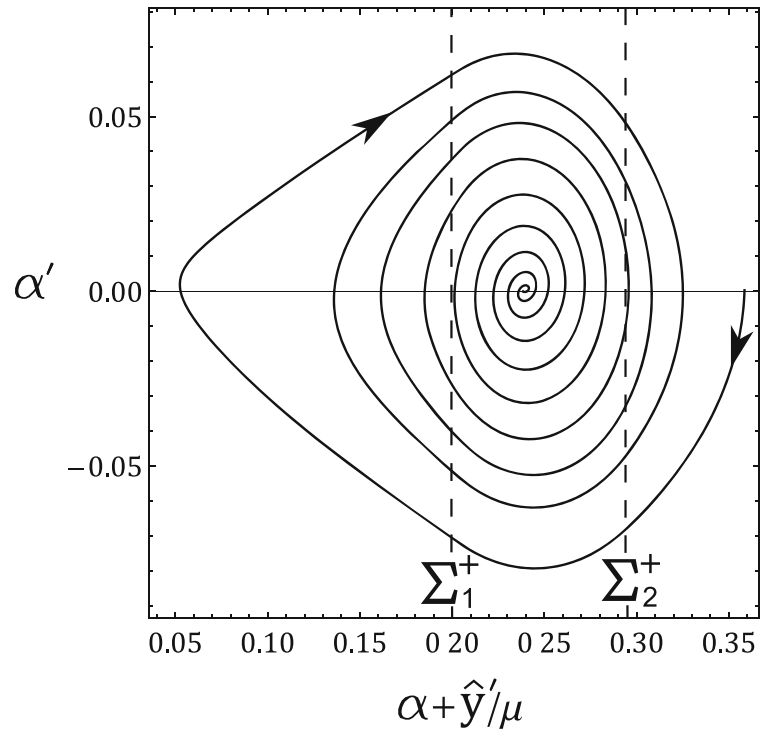

(a) $\mu=0.26<\mu_{R B}=0.304$

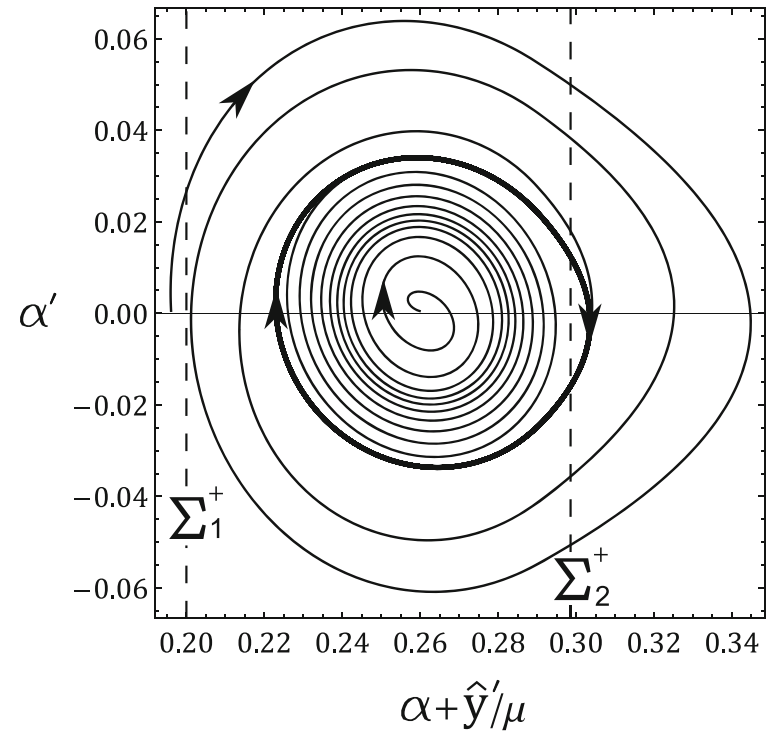

(b) $\mu=0.31>\mu_{R B}=0.304$

Fig. 8 Typical trajectories $\mathbf{a}$ before and $\mathbf{b}$ after the rapid bifurcation

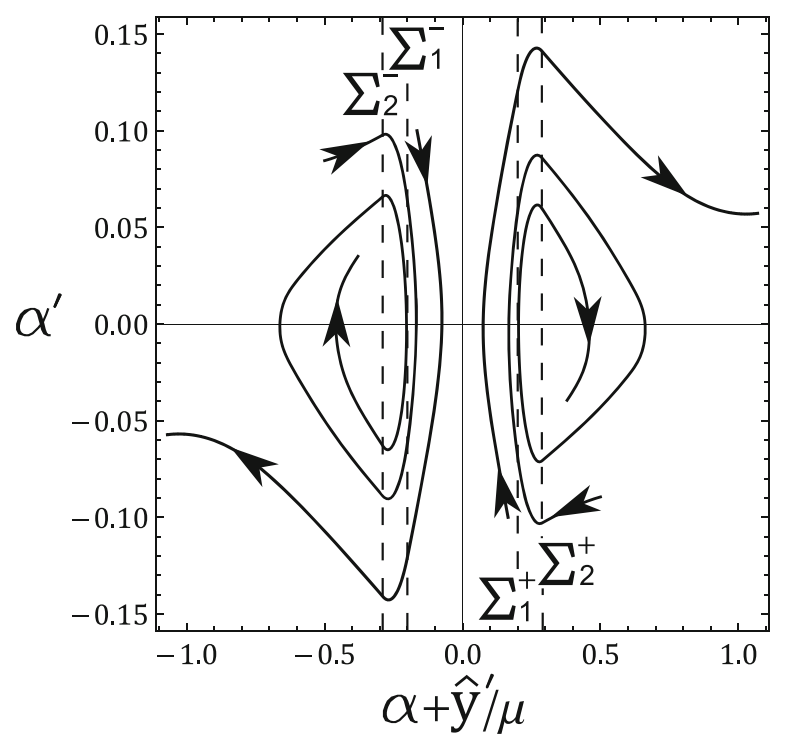

(a) $\mu=0.35<\mu_{2}=0.391$

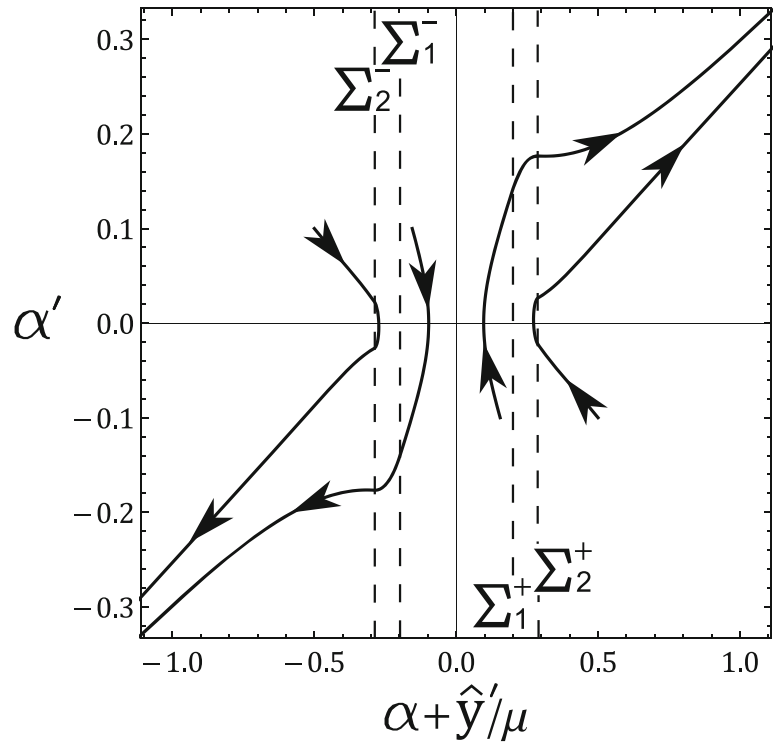

(b) $\mu=0.4>\mu_{2}=0.391$

Fig. 9 Typical trajectories a before and $\mathbf{b}$ after the second-border collision 


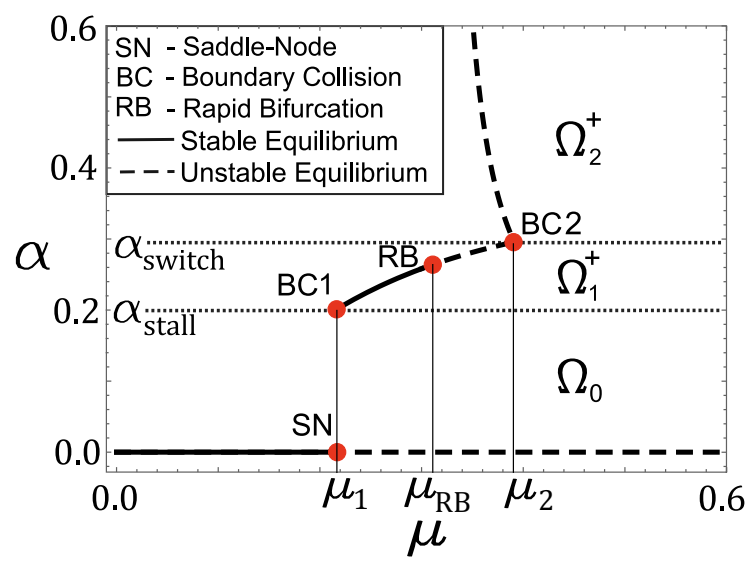

Fig. 10 Bifurcation diagram of the model with symmetric NACA 0012 profile parameters

We observe that

$$
\begin{aligned}
& a_{4}(0, \mu)<a_{2}(0, \mu) \\
& \quad=a_{4}(0, \mu)+p_{2} p_{3} c_{0} \mu+p_{1} p_{2}+1,
\end{aligned}
$$

and $a_{2}(0, \mu), a_{4}(0, \mu)$ are quadratic in $\mu$ with negative leading coefficients. Hence $a_{4}(0, \mu)=0$ has the smallest positive root (where equilibrium $\boldsymbol{E}_{0}$ loses its stability) and is given by (see Eq. (37))

$\mu_{1}=\sqrt{\frac{p_{4}}{c_{0}}}=0.215$.

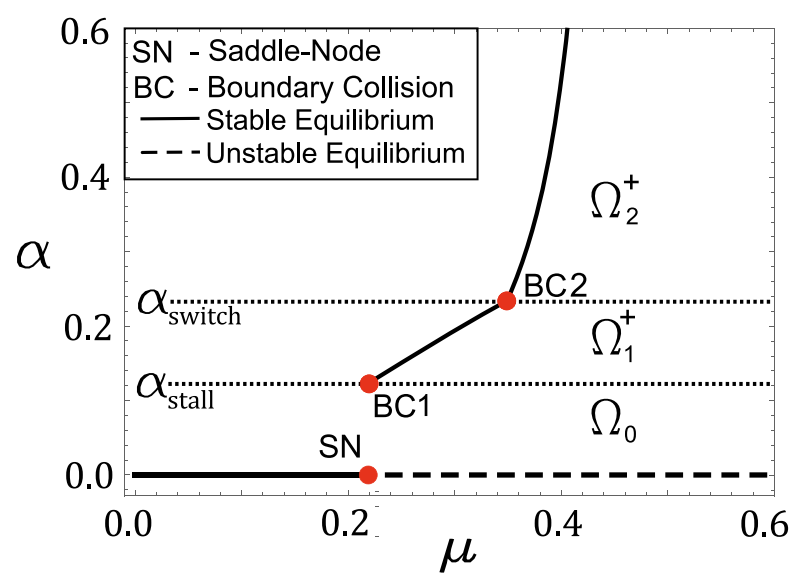

(a) $\mathrm{NACA} 0009$
For equilibria $\boldsymbol{E}_{1}^{ \pm}$, it can easily be seen that expressions $a_{2}(1, \mu), a_{4}(1, \mu)$ are positive for $\mu \geq 0$. Therefore we need to find the smallest positive root of

$$
\begin{aligned}
\Delta_{1}(1, \mu)= & 0.2025-0.1007 \mu=0, \\
\Delta_{3}(1, \mu)= & 0.0044-0.0031 \mu-0.0779 \mu^{2}+0.0993 \mu^{3} \\
& +0.3259 \mu^{4}+0.7009 \mu^{5}=0 .
\end{aligned}
$$

The smallest positive root of Eq. (57) is

$\mu_{\mathrm{RB}}=0.304$.

At $\mu=\mu_{\mathrm{RB}}$ equilibria $\boldsymbol{E}_{1}^{ \pm}$undergo a rapid bifurcation (see Sect. 7).

For equilibria $\boldsymbol{E}_{2}^{ \pm}$the Hurwitz determinants $\Delta_{1}(2, \mu)$, $\Delta_{3}(2, \mu)$ are positive for $\mu \geq 0$ (similarly as in the case of $\left.\boldsymbol{E}_{0}\right)$. From equations $a_{2}(2, \mu)=0, a_{4}(2, \mu)=0$ the smallest positive root is

$\mu_{A}=0.321$.

To summarize, equilibrium $\boldsymbol{E}_{0}$ is admissible and asymptotically stable for $\mu \in\left[0, \mu_{1}\right)$, stable at $\mu=\mu_{1}$ and unstable for $\mu>\mu_{1}$. Equilibria $\boldsymbol{E}_{1}^{ \pm}$are admissible and asymptotically stable for $\mu \in\left[\mu_{1}, \mu_{\mathrm{RB}}\right)$, stable at $\mu=\mu_{\mathrm{RB}}$ and unstable for $\in\left(\mu_{\mathrm{RB}}, \mu_{2}\right]$. Equilibria $\boldsymbol{E}_{2}^{ \pm}$ are admissible and unstable for $\mu \in\left(\mu_{A}, \mu_{2}\right]$.

\section{Classical and discontinuity-induced bifurcations}

The results in this section pertain to structural and aerodynamic parameters of the model with a NACA

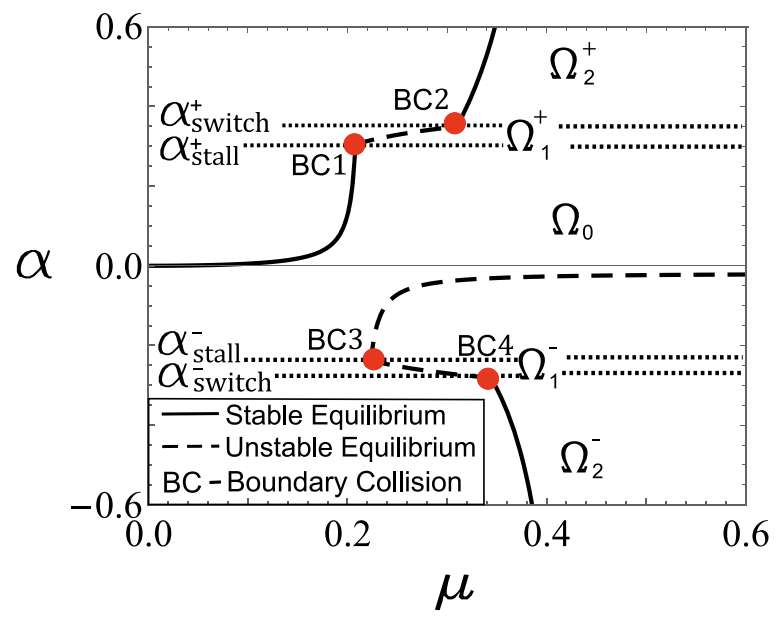

(b) NACA 23012

Fig. 11 Bifurcation diagrams of admissible equilibria of the model with symmetric NACA 0009 and asymmetric NACA 23012 profile parameters 


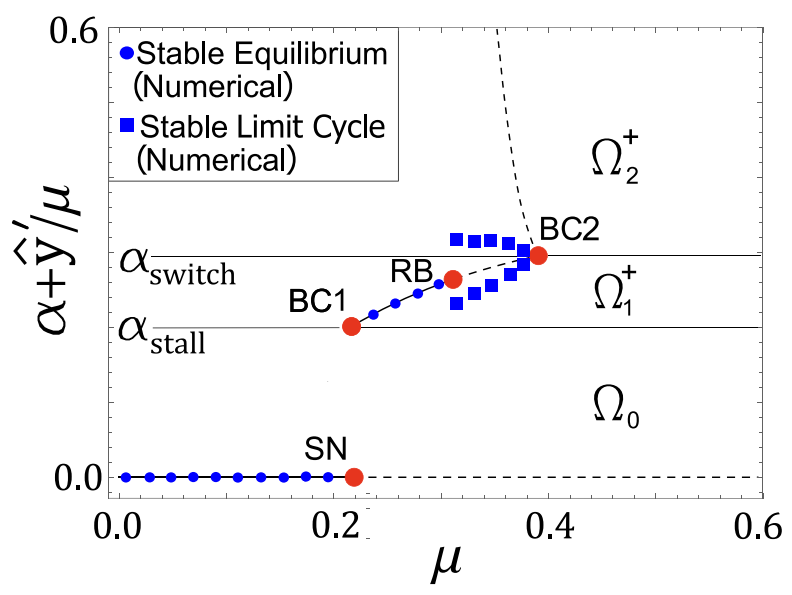

(a) NACA 0012

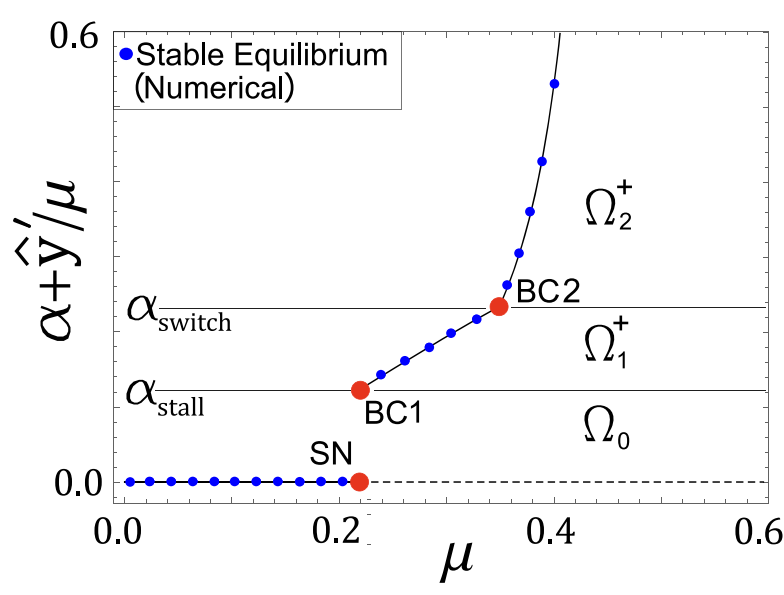

(b) NACA 0009

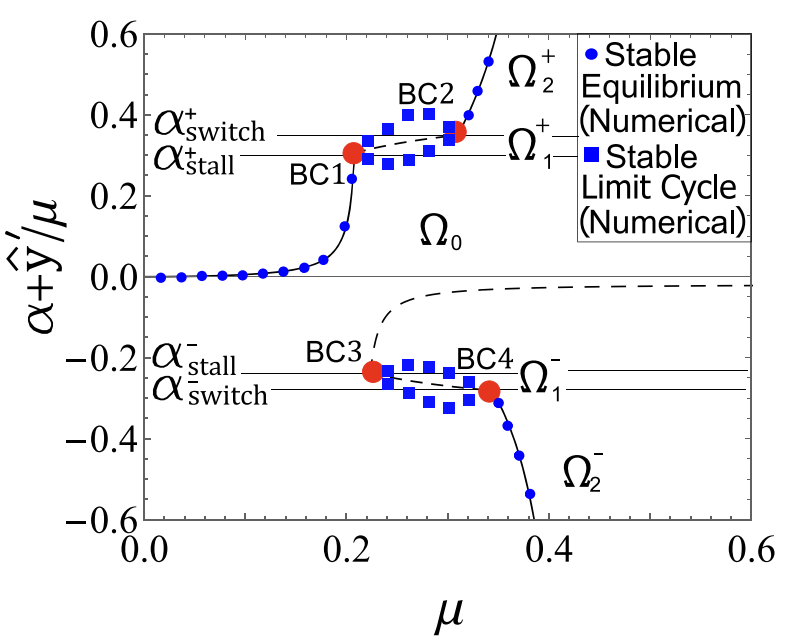

(c) NACA 23012

Fig. 12 Numerical bifurcation diagrams of the model with symmetric NACA 0012, NACA 0009 and asymmetric NACA 23012 profile parameters

0012 profile (Tables 1 and 2). This profile is symmetric; therefore, we only state the results for equilibria with the + superscript. In piecewise linear aeroelastic system (17)-(21) discontinuity-induced bifurcations $[45,46,48,49]$ are also observed in addition to their classical counterparts: border collision and rapid bifurcation.

Border collision is a boundary equilibrium bifurcation where virtual equilibrium points become admissible. The rapid bifurcation is the abrupt appearance of stable periodic oscillations $[50,51]$. The rapid bifurcation of a piecewise linear system is also called a focuscenter-limit cycle bifurcation, describing its bifurcation scenario [52]. As shown in Sect. 6, loss of stability of equilibria corresponds to the values of dimensionless freestream velocity

$0<\mu_{1}<\mu_{\mathrm{RB}}<\mu_{A}$,

where $\mu_{1}=0.215, \mu_{\mathrm{RB}}=0.304$ and $\mu_{A}=0.321$.

The first classical bifurcation occurs when equilibrium $\boldsymbol{E}_{0}$ loses stability at $\mu_{1}=0.215$. At this bifurcation point the roots of the characteristic equation $R_{0}\left(\lambda, \mu_{1}\right)=0$ (Eq. (46)) are

$\lambda_{1}=0, \lambda_{2}=-0.059, \lambda_{3}=-0.081-0.996 i$,

$\lambda_{4}=-0.081+0.996 i$.

From these eigenvalues we conclude that equilibrium $\boldsymbol{E}_{0}$ loses its stability by undergoing a saddle-node bifurcation. 


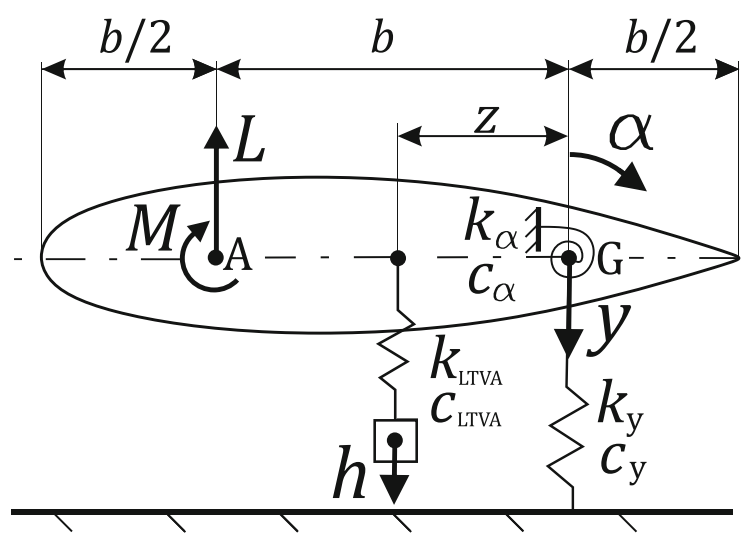

Fig. 13 The 2-DOF pitch-plunge model with LTVA

The second bifurcation of system (17)-(21) is a border collision (BC) of equilibrium $\boldsymbol{E}_{1}^{+}$at $\mu_{1}=0.215$. This border collision occurs simultaneously with the saddle node bifurcation of equilibrium $\boldsymbol{E}_{0}$. At $\mu=\mu_{1}$ the virtual equilibrium $\boldsymbol{E}_{1}^{+}$becomes admissible in the $\Omega_{1}^{+}$region by crossing the switching plane $\Sigma_{1}^{+}$. The typical trajectories of system (17)-(21) before and after the first-border collision bifurcation $\left(\mu_{1}=0.215\right)$ are illustrated in Fig. 7.

The third bifurcation is a rapid bifurcation [53] of equilibrium $\boldsymbol{E}_{1}^{+}$at $\mu_{\mathrm{RB}}=0.304$. At the rapid bifurcation point the roots of the characteristic equation $R_{1}\left(\lambda, \mu_{\mathrm{RB}}\right)=0$ (Eq. (46)) are:

$\lambda_{1}=1.023 i, \lambda_{2}=-1.023 i$,

$\lambda_{3}=-0.086-0.926 i, \lambda_{4}=-0.086+0.926 i$.

At this point the pair of complex conjugate roots $\lambda_{1}, \lambda_{2}$ of the characteristic equation $R_{1}(\lambda, \mu)=0$ crosses the imaginary axis with positive speed, similarly to the Hopf bifurcation for smooth systems. Figure 8 demonstrates that at the rapid bifurcation the stable spiral equilibrium $\boldsymbol{E}_{1}^{+}$becomes unstable, and a finite-amplitude limit cycle is born. We note that the limit cycle amplitude decreases by increasing the bifurcation parameter $\mu$.

The limit cycle oscillations are the consequence of the piecewise linear nature of the system as follows. For $\mu \in\left[\mu_{\mathrm{RB}}, \mu_{A}\right]=[0.304,0.321]$ aeroelastic systems (17)-(21) have only unstable admissible equilibria. The stable virtual equilibria $\boldsymbol{E}_{2}^{ \pm}$influence the global dynamics by "creating" a limit cycle oscillation after the rapid bifurcation. For $\mu \in\left(\mu_{A}, \mu_{2}\right]=$ $(0.321,0.391]$ aeroelastic system (17)-(21) has only unstable admissible equilibrium points; therefore, the limit cycle is created by unstable equilibria. The limit cycle oscillations exist for $\mu \in\left[\mu_{\mathrm{RB}}, \mu_{2}\right)=$ $[0.304,0.391)$. At $\mu_{2}=0.391$ two branches of unstable equilibria intersect. At this point, the amplitude of the limit cycle becomes 0 .

The fourth bifurcation of system (17)-(21) is a border collision (BC) of equilibria $\boldsymbol{E}_{1}^{+}$and $\boldsymbol{E}_{2}^{+}$at $\mu_{2}=$ 0.391 . At this border collision the admissible equilibria $\boldsymbol{E}_{1}^{+}$and $\boldsymbol{E}_{2}^{+}$become virtual by simultaneously crossing the switching plane $\Sigma_{2}^{+}$. This type of border collision (when two admissible equilibria simultaneously cross the same switching plane and become virtual) is also called as a nonsmooth fold bifurcation of piecewise systems [45].Typical trajectories of system (17 )-(21) before and after the second-border collision bifurcation $\left(\mu_{2}=0.391\right)$ are illustrated in Fig. 9 .

The bifurcation diagram of system (17)-(21) is shown in Fig. 10, where the solid line indicates the stable, and the dashed line the unstable equilibrium points. The bifurcation points are denoted by dots.

Even though the derivation here does not include formulae for the NACA 0009 and NACA 23012 profiles, we nevertheless show their bifurcation diagrams in Fig. 11.

The above results were confirmed numerically using Mathematica. The built-in event location method with a maximum step size of 0.001 was used to solve the piecewise linear system. For a given $\mu$, a onedimensional Poincaré section (the set of points for which $\alpha^{\prime}=0$ ) was computed, and these sections were spliced together to obtain the diagrams as a function of $\mu$ (see Fig. 12). The numerical results were overlaid on top of the bifurcation diagrams of Figs. 10 and 11.

\section{Stall flutter reduction with a linear tuned vibration absorber}

Stall flutter is a dynamic aeroelastic instability resulting in unwanted oscillatory loads on the structure [54]. Stall flutter of aircraft wings is caused by nonlinear aerodynamic characteristics at very high angles of attack, when a partial or complete separation of the fluid flow from the airfoil occurs periodically during the oscillation $[3,55]$. Static divergence can cause asymmetric stall flutter oscillations around nonzero pitch angles $[17,56]$. Piecewise linear model (17)-(21) captures the stall flutter aeroelastic phenomenon influenced by the static divergence. 


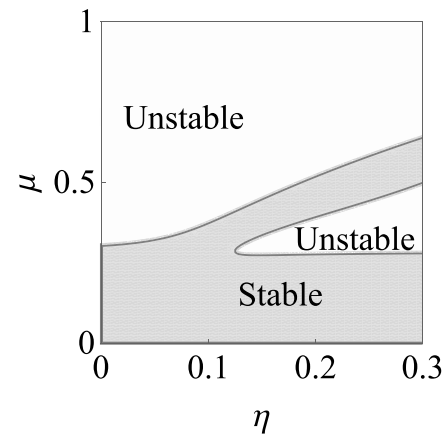

(a) $\xi=0.05, \zeta=0$

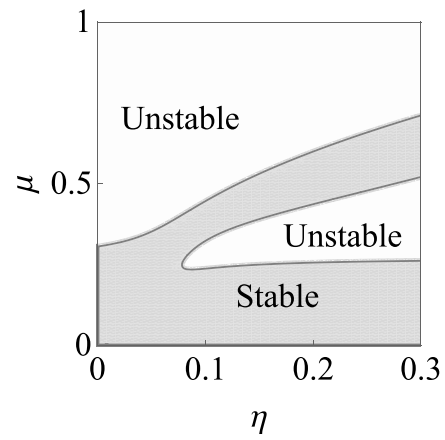

(d) $\xi=0.05, \zeta=0.01$

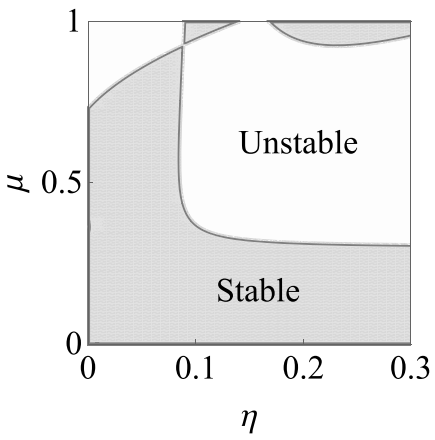

(g) $\xi=0.05, \zeta=0.05$

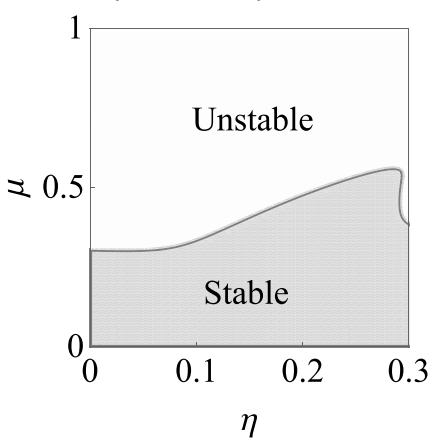

(j) $\xi=0.05, \zeta=-0.01$

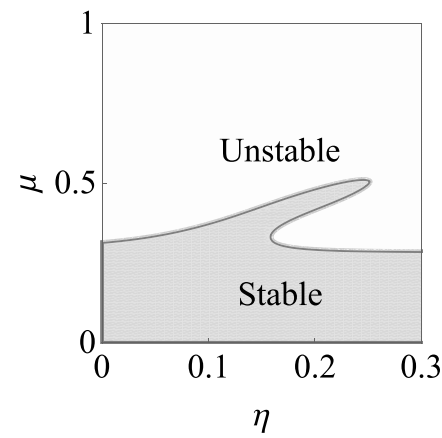

(b) $\xi=0.4, \zeta=0$

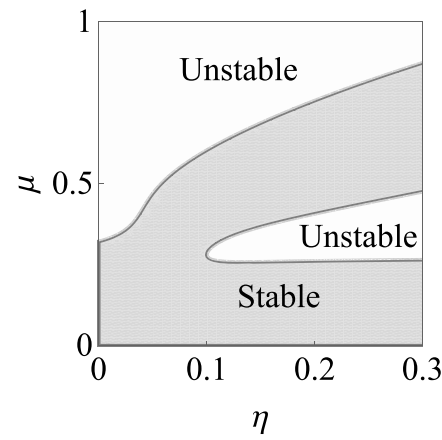

(e) $\xi=0.2, \zeta=0.01$

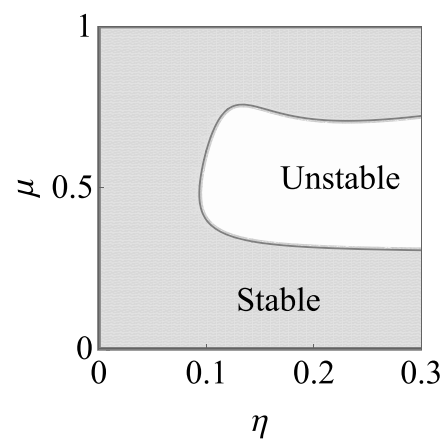

(h) $\xi=0.2, \zeta=0.05$

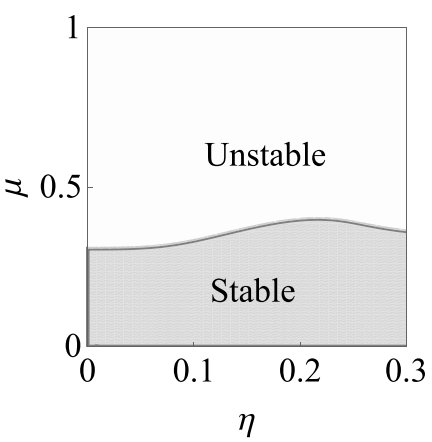

(k) $\xi=0.2, \zeta=-0.01$

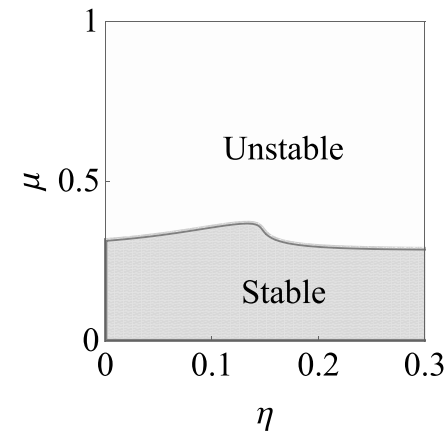

(c) $\xi=0.5, \zeta=0$

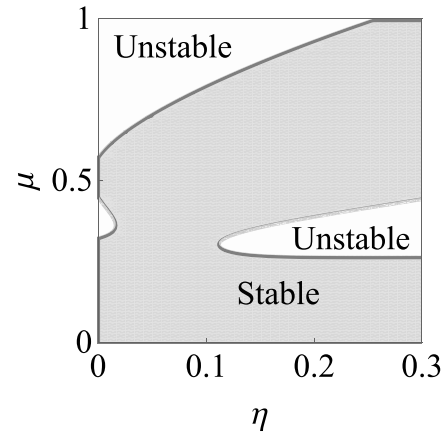

(f) $\xi=0.5, \zeta=0.01$

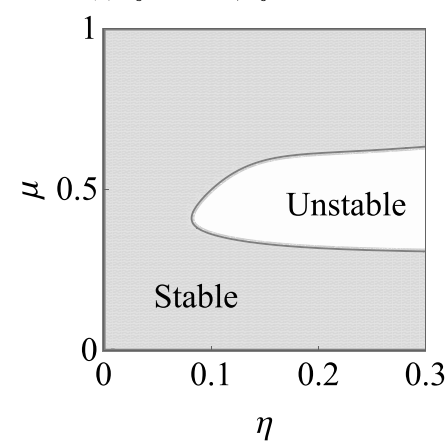

(i) $\xi=0.5, \zeta=0.05$

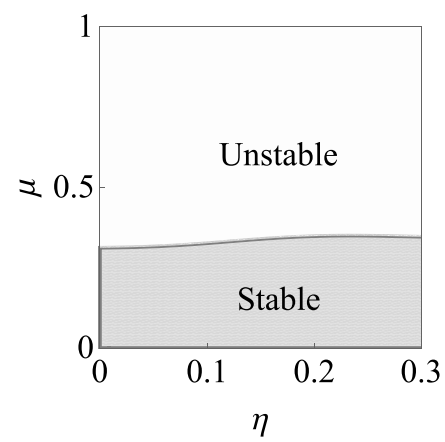

(I) $\xi=0.5, \zeta=-0.01$

Fig. 14 Stable and unstable regions of equilibria $\boldsymbol{F}_{1}^{ \pm}$in the parameter space $(\eta, \mu)$, for aerodynamic parameters of the NACA 0012 profile 


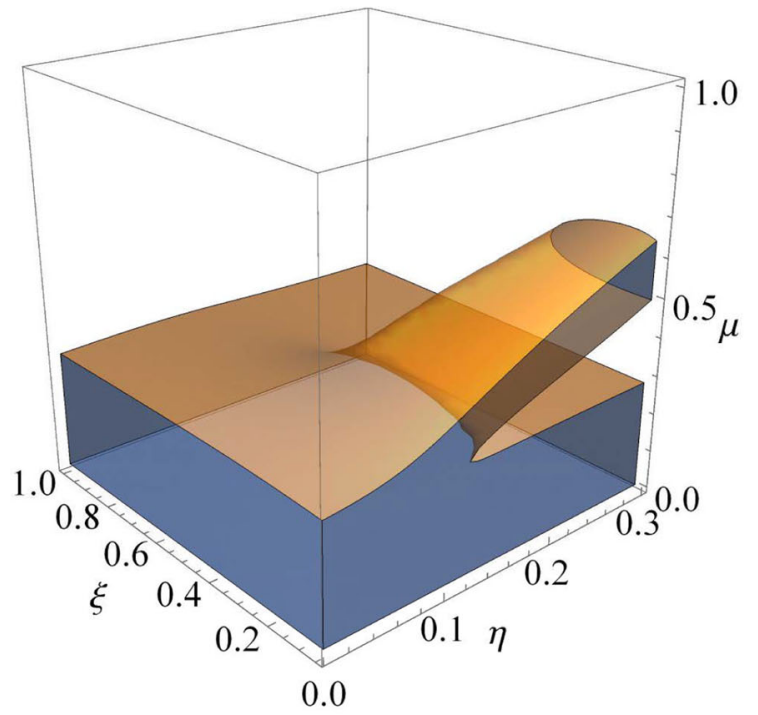

(a) $\zeta=0$

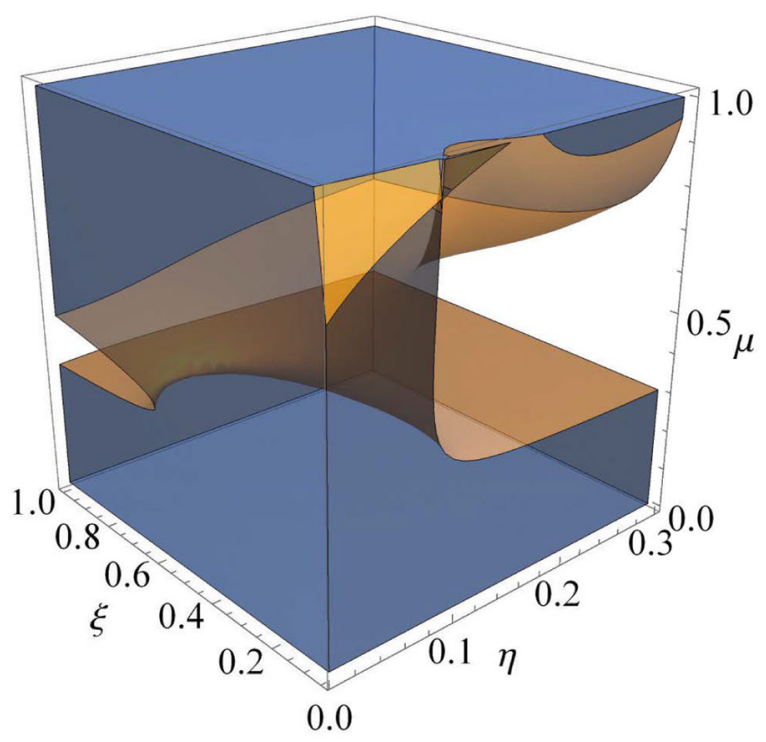

(c) $\zeta=0.05$.

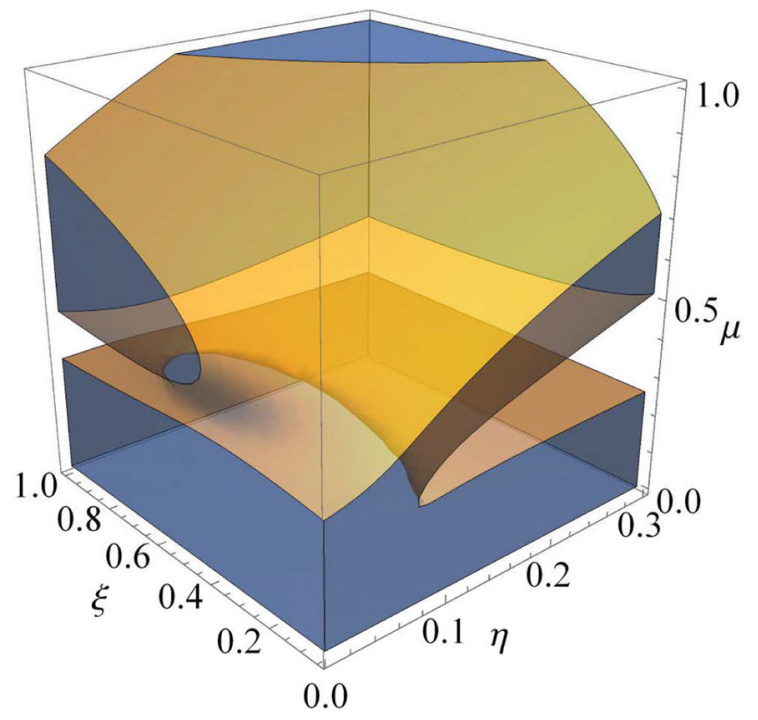

(b) $\zeta=0.01$

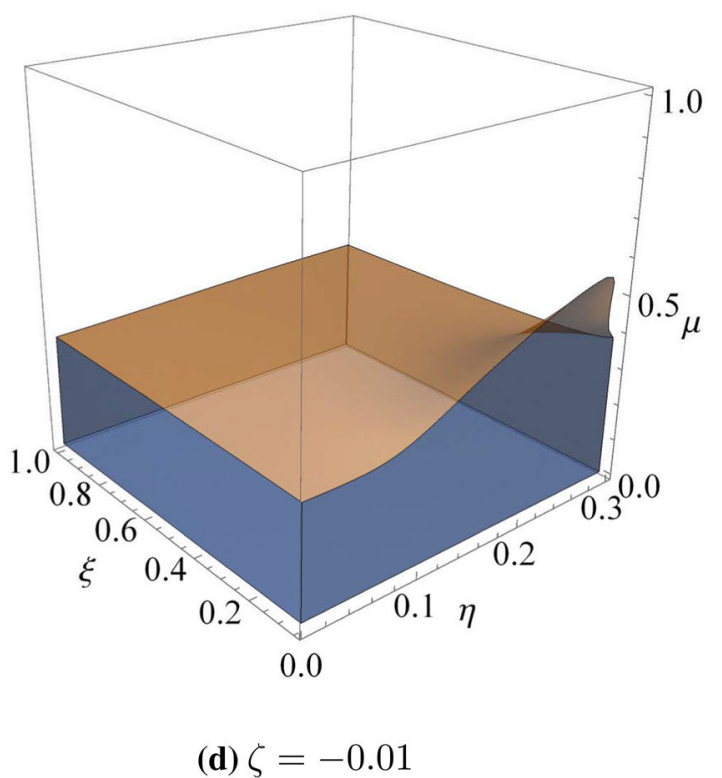

Fig. 15 Regions of stable of equilibria $\boldsymbol{F}_{1}^{ \pm}$in the parameter space $(\xi, \eta, \mu)$ for aerodynamic parameters of the NACA 0012 profile (Table 2)

Increasing the nondimensional freestream velocity above the rapid bifurcation point (for NACA 0012 profile aerodynamic parameters) the system starts to oscillate. To eliminate these stall flutter oscillations we applied a linear tuned vibration absorber (LTVA) to the primary system, see Fig. 13. The absorber is attached along the mid-chord of the airfoil at distance $z$ from the center of gravity (G). It is composed of a mass $m_{l t v a}$, a spring of linear stiffness $k_{l t v a}$ and a linear damper $c_{l t v a}$. The equations of motion of the aeroelastic system with the absorber are

$$
\begin{aligned}
& m \ddot{y}+c_{y} \dot{y}+k_{y} y-f(y, \dot{y}, \alpha, \dot{\alpha}, h, \dot{h})=-L\left(C_{l}\left(\alpha_{\mathrm{eff}}\right)\right), \\
& I_{\mathrm{cg}} \ddot{\alpha}+c_{\alpha} \dot{\alpha}+k_{\alpha} \alpha+z f(y, \dot{y}, \alpha, \dot{\alpha}, h, \dot{h}) \\
& \quad=M\left(C_{l}\left(\alpha_{\mathrm{eff}}\right)\right), \\
& m_{\text {ltva }} \ddot{h}+f(y, \dot{y}, \alpha, \dot{\alpha}, h, \dot{h})=0,
\end{aligned}
$$




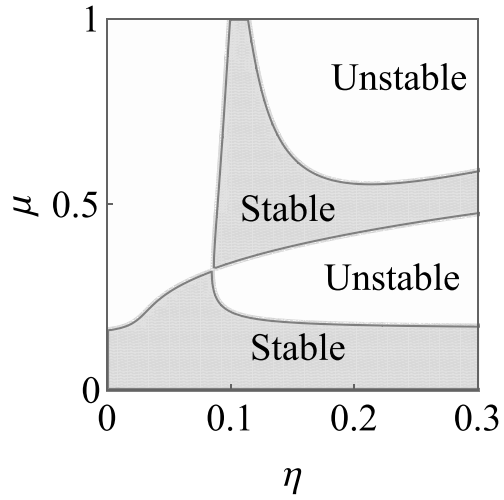

(a) $\xi=0.05$

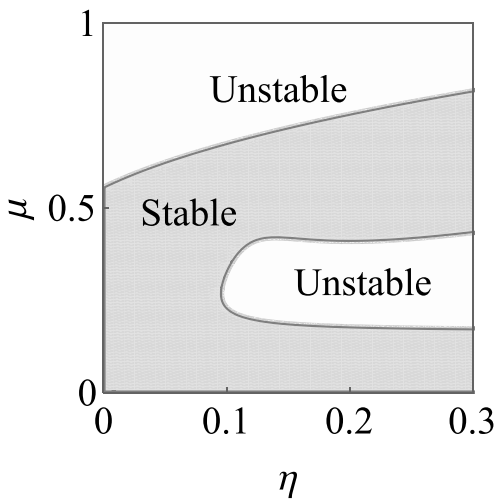

(b) $\xi=0.2$

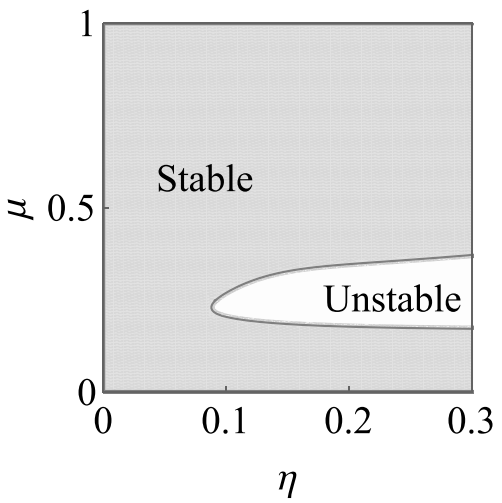

(c) $\xi=0.5$

Fig. 16 Stable and unstable regions of equilibrium $\boldsymbol{F}_{1}^{+}$in the parameter space $(\eta, \mu)$, for aerodynamic parameters of the NACA 23012 profile $(\zeta=0.05)$

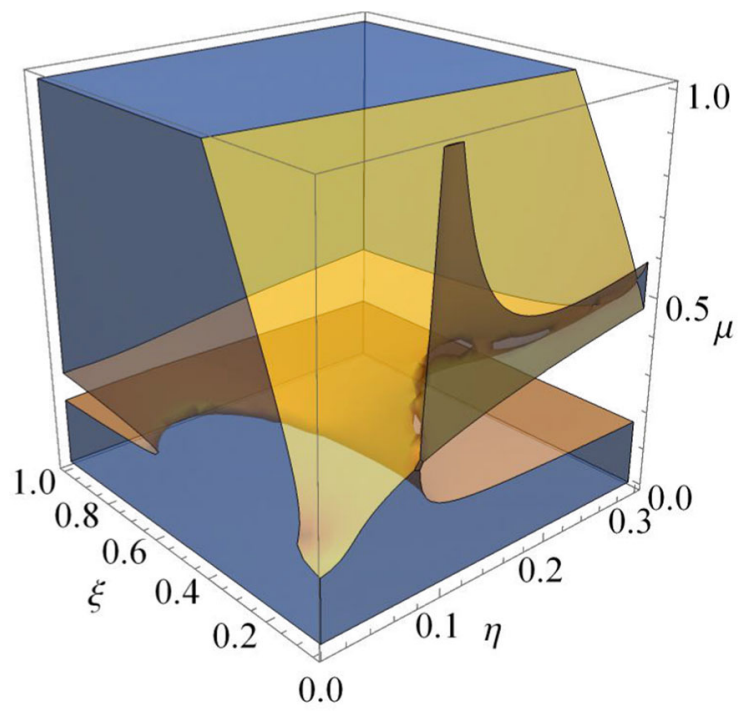

Fig. 17 Regions of stable of equilibrium $\boldsymbol{F}_{1}^{+}$in the parameter space $(\xi, \eta, \mu)$, for aerodynamic parameters of the NACA 23012 profile $(\zeta=0.05)$

where

$$
\begin{aligned}
f(y, \dot{y}, \alpha, \dot{\alpha}, h, \dot{h})= & c_{\text {ltva }}(\dot{h}-(\dot{y}-z \dot{\alpha})) \\
& +k_{\text {ltva }}(h-(y-z \alpha)) .
\end{aligned}
$$

Equations (63) and (64) are nondimensionalized by a length scale $\mathcal{L}$, a timescale $\mathcal{T}$ and a nondimensional freestream velocity $\mu>0$, given in Eq. (7). We define the damping ratio $\xi=c_{l t v a} / c_{y}$, the stiffness ratio $\eta=k_{\text {ltva }} / k_{y}$ and the mass ratio $\varepsilon=m_{\text {ltva }} / m$. The nondimensional distance of the absorber from the center of gravity is defined as $\zeta=z / \mathcal{L}$. The nondi- mensional displacement of the absorber is denoted by $\hat{h}=h / \mathcal{L}$. The nondimensional equation will be the following

$$
\begin{aligned}
& \hat{y}^{\prime \prime}+p_{1} \hat{y}^{\prime}+\hat{y}-\hat{f}\left(\hat{y}, \hat{y}^{\prime}, \alpha, \alpha^{\prime}, \hat{h}, \hat{h}^{\prime}\right) \\
& \quad=-p_{2} \mu^{2} C_{l}\left(\alpha+\frac{1}{\mu} \hat{y}^{\prime}\right), \\
& \alpha^{\prime \prime}+p_{3} \alpha^{\prime}+p_{4} \alpha+w \zeta p_{4} \hat{f}\left(\hat{y}, \hat{y}^{\prime}, \alpha, \alpha^{\prime}, \hat{h}, \hat{h}^{\prime}\right) \\
& \quad=\mu^{2} C_{l}\left(\alpha+\frac{1}{\mu} \hat{y}^{\prime}\right), \\
& \varepsilon \hat{h}^{\prime \prime}+\hat{f}\left(\hat{y}, \hat{y}^{\prime}, \alpha, \alpha^{\prime}, \hat{h}, \hat{h}^{\prime}\right)=0,
\end{aligned}
$$

where

$$
\begin{aligned}
\hat{f}\left(\hat{y}, \hat{y}^{\prime}, \alpha, \alpha^{\prime}, \hat{h}, \hat{h}^{\prime}\right)= & \xi p_{1}\left(\hat{h}^{\prime}-\left(\hat{y}^{\prime}-\zeta \alpha^{\prime}\right)\right) \\
& +\eta(\hat{h}-(\hat{y}-\zeta \alpha)),
\end{aligned}
$$

and the nondimensional coupling ratio is

$w=\frac{k_{y}}{k_{\alpha}} \mathcal{L}^{2}=\frac{k_{y}}{k_{\alpha}} \frac{I_{\mathrm{cg}}}{\rho b^{2} S}$.

We extend the state vector as

$\boldsymbol{x}=\left[\begin{array}{c}\hat{y} \\ \hat{y}^{\prime} \\ \alpha \\ \alpha^{\prime} \\ \hat{h} \\ \hat{h}^{\prime}\end{array}\right]$. 


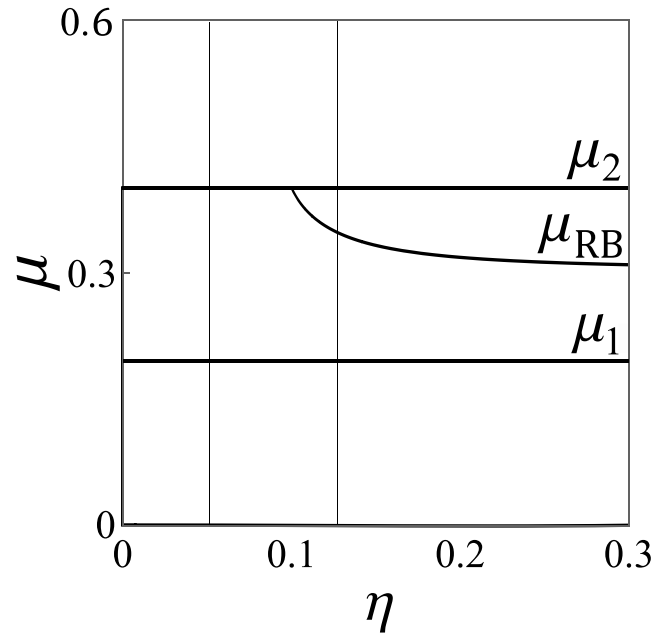

(a) Bifurcation values $\mu_{1}, \mu_{R B}, \mu_{2}$ as the function of $\eta$ (thin vertical lines indicate $\eta=0.05$ and $\eta=0.12$ )

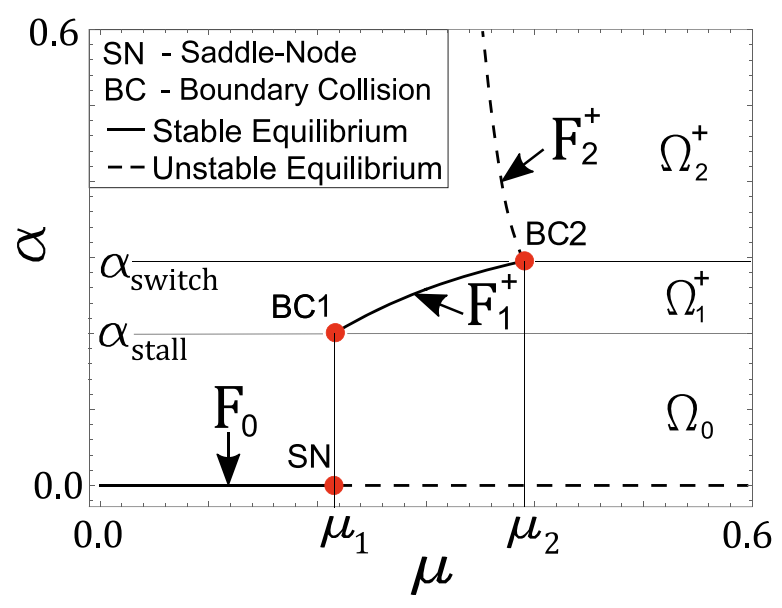

(b) Bifurcation diagram for $\eta=0.05$

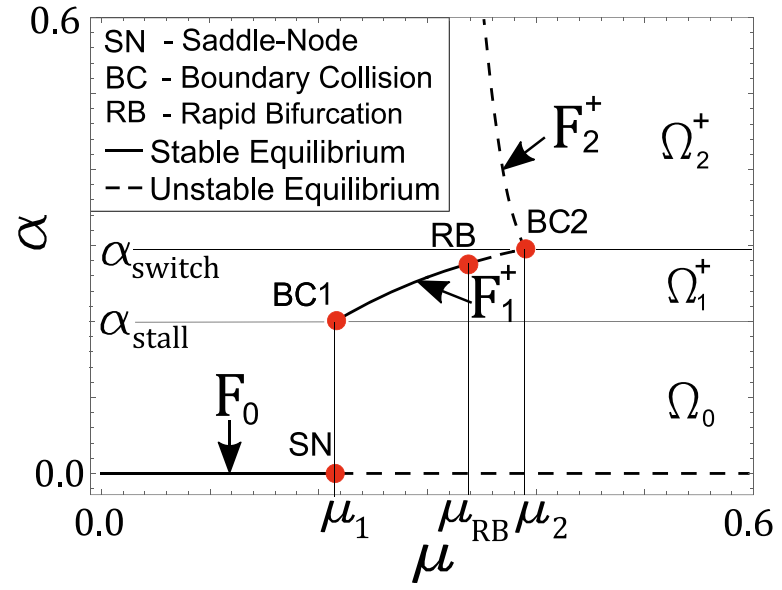

(c) Bifurcation diagram for $\eta=0.12$

Fig. 18 Effect of the absorber on the bifurcation points with parameters listed in Eq. (101)

We define the new system matrices

$$
\boldsymbol{Q}_{k}=\left[\begin{array}{cccccc}
0 & 1 & 0 & 0 & 0 & 0 \\
-1-\eta-\left(p_{1}+p_{2} \mu c_{k}\right)-\xi p_{1} & -p_{2} \mu^{2} c_{k}+\zeta \eta & \zeta \xi p_{1} & \eta & \xi p_{1} \\
0 & 0 & 0 & 1 & 0 & 0 \\
w \zeta \eta p_{4} & \mu c_{k}+w \zeta \xi p_{1} p_{4} & -\left(p_{4}-\mu^{2} c_{k}\right)-w \zeta^{2} \eta p_{4} & -p_{3}-w \zeta^{2} \xi p_{1} p_{4} & -w \zeta \eta p_{4} & -w \zeta \xi p_{1} p_{4} \\
0 & 0 & 0 & 0 & 0 & 1 \\
\frac{\eta}{\varepsilon} & \frac{\xi p_{1}}{\varepsilon} & -\frac{\zeta \eta}{\varepsilon} & -\frac{\zeta \xi p_{1}}{\varepsilon} & -\frac{\eta}{\varepsilon} & -\frac{\xi p_{1}}{\varepsilon}
\end{array}\right], k=0,1,2
$$




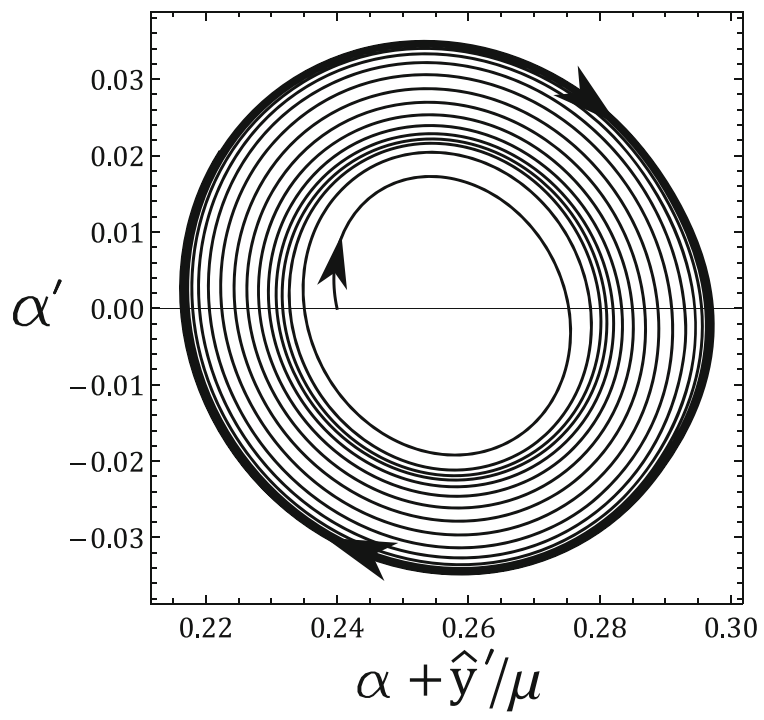

(a) Without LTVA

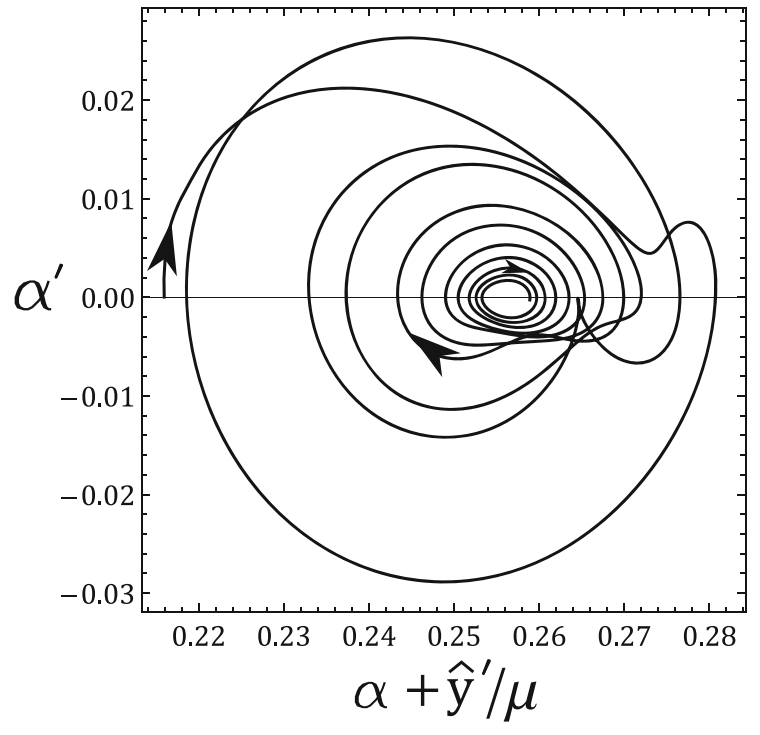

(b) With LTVA

Fig. 19 Suppression of limit cycle oscillation at $\mu=0.31>\mu_{\mathrm{RB}}$ (aerodynamic parameters: NACA 0012 profile, absorber parameters: $\varepsilon=0.1, \xi=0.2, \eta=0.05, \zeta=0.05$ )

and translation vectors

$\boldsymbol{r}_{k}=\left[\begin{array}{c}0 \\ -p_{2} \mu^{2} d_{k} \\ 0 \\ \mu^{2} d_{k} \\ 0 \\ 0\end{array}\right], \quad k=1,2$

The equations of the extended system are

$\dot{\boldsymbol{x}}=\boldsymbol{Q}_{2} \boldsymbol{x}-\boldsymbol{r}_{2}, \quad \boldsymbol{x} \in \Omega_{2}^{-} \cup \Sigma_{2}^{-}$,

$\dot{\boldsymbol{x}}=Q_{1} \boldsymbol{x}-\boldsymbol{r}_{1}, \quad \boldsymbol{x} \in \Sigma_{2}^{-} \cup \Omega_{1}^{-} \cup \Sigma_{1}^{-}$,

$\dot{\boldsymbol{x}}=Q_{0} \boldsymbol{x}, \quad \boldsymbol{x} \in \Sigma_{1}^{-} \cup \Omega_{0} \cup \Sigma_{1}^{+}$,

$\dot{\boldsymbol{x}}=\boldsymbol{Q}_{1} \boldsymbol{x}+\boldsymbol{r}_{1}, \quad \boldsymbol{x} \in \Sigma_{1}^{+} \cup \Omega_{1}^{+} \cup \Sigma_{2}^{+}$,

$\dot{\boldsymbol{x}}=\boldsymbol{Q}_{2} \boldsymbol{x}+\boldsymbol{r}_{2}, \quad \boldsymbol{x} \in \Sigma_{2}^{+} \cup \Omega_{2}^{+}$,

where the domains of subsystems (71)-(75) are given by

$$
\begin{aligned}
& \Omega_{2}^{-}:=\left\{x \in \mathbb{R}^{6}: \alpha+\frac{\hat{y}^{\prime}}{\mu} \in\left(-\infty,-\alpha_{\text {switch }}\right)\right\} \\
& \Omega_{1}^{-}:=\left\{x \in \mathbb{R}^{6}: \alpha+\frac{\hat{y}^{\prime}}{\mu} \in\left(-\alpha_{\text {switch }},-\alpha_{\text {stall }}\right)\right\} \\
& \Omega_{0}:=\left\{x \in \mathbb{R}^{6}: \alpha+\frac{\hat{y}^{\prime}}{\mu} \in\left(-\alpha_{\text {stall }}, \alpha_{\text {stall }}\right)\right\} \\
& \Omega_{1}^{+}:=\left\{x \in \mathbb{R}^{6}: \alpha+\frac{\hat{y}^{\prime}}{\mu} \in\left(\alpha_{\text {stall }}, \alpha_{\text {switch }}\right)\right\}
\end{aligned}
$$

$\Omega_{2}^{+}:=\left\{\boldsymbol{x} \in \mathbb{R}^{6}: \alpha+\frac{\hat{y}^{\prime}}{\mu} \in\left(\alpha_{\text {switch }}, \infty\right)\right\}$.

These domains are separated by the switching planes defined by

$\Sigma_{1}^{ \pm}:=\left\{\boldsymbol{x} \in \mathbb{R}^{6}: \alpha+\frac{\hat{y}^{\prime}}{\mu}= \pm \alpha_{\text {stall }}\right\}$,

$\Sigma_{2}^{ \pm}:=\left\{x \in \mathbb{R}^{6}: \alpha+\frac{\hat{y}^{\prime}}{\mu}= \pm \alpha_{\text {switch }}\right\}$.

With these pairwise disjoint sets, the full state space $\mathbb{R}^{6}$ can be decomposed as

$$
\begin{aligned}
\mathbb{R}^{6}= & \Omega_{2}^{-} \cup \Sigma_{2}^{-} \cup \Omega_{1}^{-} \cup \Sigma_{1}^{-} \cup \Omega_{0} \\
& \cup \Sigma_{1}^{+} \cup \Omega_{1}^{+} \cup \Sigma_{2}^{+} \cup \Omega_{2}^{+} .
\end{aligned}
$$

\subsection{Equilibrium points}

The admissible equilibrium points of subsystems (71)(75) are

$$
\begin{gathered}
\boldsymbol{F}_{0}=\mathbf{0}, \quad \boldsymbol{F}_{0} \in \Sigma_{1}^{-} \cup \Omega_{0} \cup \Sigma_{1}^{+}, \\
\boldsymbol{F}_{1}^{ \pm}=\mp \boldsymbol{Q}_{1}^{-1} \boldsymbol{r}_{1}=\left[\begin{array}{c}
\boldsymbol{E}_{1}^{ \pm} \\
\pm \frac{d_{1}\left(p_{2} p_{4}+\zeta\right) \mu^{2}}{c_{1} \mu^{2}-p_{4}} \\
0
\end{array}\right], \\
\boldsymbol{F}_{1}^{ \pm} \in \Sigma_{1}^{ \pm} \cup \Omega_{1}^{ \pm} \cup \Sigma_{2}^{ \pm},
\end{gathered}
$$




$$
\begin{aligned}
\boldsymbol{F}_{2}^{ \pm}= & \mp \boldsymbol{Q}_{2}^{-1} \boldsymbol{r}_{2}=\left[\begin{array}{c}
\boldsymbol{E}_{2}^{ \pm} \\
\pm \frac{d_{2}\left(p_{2} p_{4}+\zeta\right) \mu^{2}}{c_{2} \mu^{2}-p_{4}} \\
0
\end{array}\right], \\
& \boldsymbol{F}_{2}^{ \pm} \in \Sigma_{2}^{ \pm} \cup \Omega_{2}^{ \pm} .
\end{aligned}
$$

The expression of equilibria $\boldsymbol{F}_{0}, \boldsymbol{F}_{1}^{ \pm}, \boldsymbol{F}_{2}^{ \pm}$and their range of validity (Eqs. (84)-(86)) determine the admissible ranges depending on the bifurcation parameter $\mu$ as

$\boldsymbol{F}_{\mathbf{0}}$ is always admissible for symmetric airfoil profiles, (87)

$\boldsymbol{F}_{1}^{ \pm}$is admissible if $\mu \in\left[\mu_{1}, \mu_{2}\right]$,

$\boldsymbol{F}_{2}^{ \pm}$is admissible if $\mu \in\left(\mu_{A}, \mu_{2}\right]$.

\subsection{Effects of the absorber parameters}

In this section we investigate the effects of the absorber parameters on the stability of equilibria $\boldsymbol{F}_{0}, \boldsymbol{F}_{1}^{ \pm}, \boldsymbol{F}_{2}^{ \pm}$. To reduce the number of parameters we fixed the mass ratio $\varepsilon=0.1$. The other absorber parameters were varied in the following intervals: $\zeta \in[-0.01,0.05]$, $\eta \in[0,0.3], \xi \in[0,1]$.

First, we investigate the asymptotic stability of these equilibria of the aeroelastic system with the LTVA. To determine the asymptotic stability of the equilibrium points, we study the characteristic polynomial of the coefficient matrices $\boldsymbol{Q}_{k}$ defined in Eq. (69)

$$
\begin{aligned}
P_{k}(\lambda, \mu)= & \lambda^{6}+q_{1}(k, \mu) \lambda^{5} \\
& +q_{2}(k, \mu) \lambda^{4}+q_{3}(k, \mu) \lambda^{3}+q_{4}(k, \mu) \lambda^{2} \\
& +q_{5}(k, \mu) \lambda+q_{6}(k, \mu),
\end{aligned}
$$

where

$$
\begin{aligned}
q_{1}(k, \mu)= & p_{3}+p_{1}+p_{2} c_{k} \mu+\xi p_{1}\left(\zeta^{2} w p_{4}+\frac{1}{\varepsilon}+1\right) \\
q_{2}(k, \mu)= & \frac{p_{4}\left(\xi p_{1}\left(c_{k} \mu p_{2}+p_{1}\right)+\varepsilon\left(\zeta \eta w\left(c_{k} \mu^{2} p_{2}+\zeta\right)+\eta+1\right)+\eta\right)}{\varepsilon} \\
& +\frac{\xi p_{1}\left(p_{3}-c_{k} \mu^{2} p_{1}\right)+c_{k} \mu\left(\eta p_{2} p_{3}-\mu(\eta+\eta \varepsilon+\varepsilon)\right)+\eta+\eta p_{1} p_{3}}{\varepsilon}, \\
q_{3}(k, \mu)= & \frac{p_{1}\left(p_{4}\left(\varepsilon\left(\zeta w\left(c_{k} \mu^{2} \xi p_{2}+\zeta(\eta+\xi)\right)+\xi+1\right)+\xi\right)-c_{k} \mu^{2}(\xi+\xi \varepsilon+\varepsilon)+\eta+\xi\right)}{\varepsilon} \\
& +\frac{c_{k} \mu\left(p_{2}\left(\eta+\zeta^{2} \eta p_{4} w \varepsilon+p_{4} \varepsilon\right)-\zeta \eta \varepsilon\right)+p_{3}\left(\xi p_{1}\left(c_{k} \mu p_{2}+p_{1}\right)+\eta+\eta \varepsilon+\varepsilon\right)}{\varepsilon}, \\
q_{4}(k, \mu)= & \frac{p_{4}\left(\xi p_{1}\left(c_{k} \mu p_{2}+p_{1}\right)+\varepsilon\left(\zeta \eta w\left(c_{k} \mu^{2} p_{2}+\zeta\right)+\eta+1\right)+\eta\right)}{\varepsilon} \\
& +\frac{\xi p_{1}\left(p_{3}-c_{k} \mu^{2} p_{1}\right)+c_{k} \mu\left(\eta p_{2} p_{3}-\mu(\eta+\eta \varepsilon+\varepsilon)\right)+\eta+\eta p_{1} p_{3}}{\varepsilon}, \\
q_{5}(k, \mu)= & \frac{\eta p_{3}+\eta p_{2} p_{4} c_{k} \mu+(\eta+\xi) p_{1}\left(p_{4}-c_{k} \mu^{2}\right)}{\varepsilon}, \\
q_{6}(k, \mu)= & \frac{\eta\left(p_{4}-\mu^{2} c_{k}\right)}{\varepsilon} .
\end{aligned}
$$


the parameters $\varepsilon, \zeta, \eta, \xi$, but this dependence is not explicitly shown to keep the notation short. Applying the Liénard-Chipart stability criterion (see Eq. (45)), the aeroelastic system with the linear tuned vibration absorber is asymptotically stable if the following conditions are fulfilled

$q_{2}(k, \mu)>0, \quad q_{4}(k, \mu)>0, \quad q_{6}(k, \mu)>0$,

$q_{1}(k, \mu)>0, \quad \Delta_{3}(k, \mu)>0, \quad \Delta_{5}(k, \mu)>0$,

where the Hurwitz determinants $\Delta_{3}(k, \mu)$ and $\Delta_{5}(k, \mu)$ are calculated according to Eq. (45).

In Sect. 6 we determined that equilibrium $\boldsymbol{E}_{0}$ is admissible and asymptotically stable for $\mu \in\left[0, \mu_{1}\right)$, stable at $\mu=\mu_{1}$ and unstable for $\mu>\mu_{1}$. The range of asymptotic stability $\mu \in\left[0, \mu_{1}\right)$ of equilibrium $\boldsymbol{F}_{0}$ is the same as that of $\boldsymbol{E}_{0}$. This is the consequence of

$q_{6}(0, \mu)=\frac{\eta}{\varepsilon} a_{4}(0, \mu)$,

which means that the zero of $q_{6}(0, \mu)\left(\mu=\mu_{1}\right)$ coincides with that of $a_{4}(0, \mu)$ at $\mu=\mu_{1}$.

Equilibria $\boldsymbol{E}_{1}^{ \pm}$are admissible and asymptotically stable for $\mu \in\left[\mu_{1}, \mu_{\mathrm{RB}}\right)$, stable at $\mu=\mu_{\mathrm{RB}}$ and unstable for $\in\left(\mu_{\mathrm{RB}}, \mu_{2}\right]$.

The range of asymptotic stability $\mu \in\left[\mu_{1}, \mu_{\mathrm{RB}}\right)$ of equilibria $\boldsymbol{F}_{1}^{ \pm}$can, however, be extended by tuning the parameters of the absorber. In other words, we can shift $\mu_{\mathrm{RB}}$ to larger $\mu$ values by appropriate absorber parameters.

The stable regions of equilibria $\boldsymbol{F}_{1}^{ \pm}$in the 2-dimensional parameter space $(\eta, \mu)$ for different $\xi$ and $\zeta$ values, determined by conditions (97)-(98), are illustrated in Fig. 14. The stable regions of equilibria $\boldsymbol{F}_{1}^{ \pm}$can also be visualized in the 3-dimensional parameter space $(\xi, \eta, \mu)$ for $\zeta \in\{0,0.01,0.05,-0.01\}$, see Fig. 15. The range of admissibility and instability $\mu \in\left(\mu_{A}, \mu_{2}\right]$ of equilibrium $\boldsymbol{F}_{2}^{ \pm}$is the same as that of $\boldsymbol{E}_{2}^{ \pm}$. This is the consequence of

$q_{6}(2, \mu)=\frac{\eta}{\varepsilon} a_{4}(2, \mu)$,

which means that the zero of $q_{6}(2, \mu)$ coincides with that of $a_{4}(2, \mu)$ at $\mu=\mu_{A}$.

Even though the derivation here does not include formulae for NACA 23012 profile, we nevertheless show the stable regions of equilibria $\boldsymbol{F}_{1}^{+}$in Figs. 16 and 17.

\subsection{Quenching oscillations with the absorber}

In this section we demonstrate that oscillations can be eliminated in certain regions of the parameter space of the absorber. Once again we use the structural and aerodynamic parameters of the model with NACA 0012 profile (Tables 1 and 2). Using absorber parameters

$\varepsilon=0.1, \xi=0.2, \zeta=0.05$,

we draw the bifurcation values $\mu_{1}, \mu_{\mathrm{RB}}, \mu_{2}$ as the functions of $\eta$ see Fig. 18a. For parameter values (101) and $\eta=0.05$ the rapid bifurcation is eliminated and the limit cycle oscillations (see Fig. 18b). For parameter values (101) and $\eta=0.12$ the value of $\mu_{\mathrm{RB}}$ is increased and the limit cycle oscillations still exist if $\mu \in\left[\mu_{\mathrm{RB}}, \mu_{2}\right.$ ) (see Fig. 18c). Typical trajectories with and without absorber are shown in Fig. 19.

\section{Conclusions}

A piecewise linear aeroelastic system with and without a tuned vibration absorber was studied. A piecewise linear model was utilized using experimental data for the lift coefficient versus the angle of attack for NACA 0012, NACA 0009 and NACA 23012 airfoil profiles. The equations of motion for the system were nondimensionalized for symmetric airfoil profiles. The nondimensional freestream velocity was introduced as the system bifurcation parameter. The equilibria of the piecewise linear aeroelastic system were determined analytically as a function of the bifurcation parameter. The admissibility conditions of the equilibria were analyzed. The stability of those equilibrium points was studied by applying the Liénard-Chipart criterion.

The bifurcation analysis found classical and discontinuity-induced bifurcations as saddle-node, border collision and rapid bifurcation. The saddle-node bifurcation corresponds to the static divergence of the aeroelastic system. The rapid bifurcation is the abrupt appearance of stable periodic stall flutter oscillations of the aeroelastic system. The bifurcation analysis showed that the pitch-plunge model with a simple piecewise linear approximation of the aerodynamic force can reproduce the complex aeroelastic phenomenon of stall flutter. Finally, the effect of absorber parameters was investigated. The stall flutter oscillations were eliminated using a well-tuned absorber. This parametric study of the linear tuned vibration absorber predicts that not only active $[32,54]$ but also passive vibration reduction methods can be applied to suppress stall flutter. 
Acknowledgements Open access funding provided by Budapest University of Technology and Economics (BME). The publication of the work reported herein has been supported by the ÚNKP-19-3 New National Excellence Program of the Ministry for Innovation and Technology of Hungary. The research reported in this paper was supported by the Higher Education Excellence Program of the Ministry of Human Capacities in the frame of the Water Sciences \& Disaster Prevention research area of BME (BME FIKP-VÍZ). The research reported in this paper has been supported by the National Research, Development and Innovation Fund (TUDFO/51757/2019-ITM, Thematic Excellence Program).

Funding Open access funding provided by Budapest University of Technology and Economics (BME).

\section{Compliance with ethical standards}

Conflict of interest The authors declare that they have no conflicts of interest.

Open Access This article is licensed under a Creative Commons Attribution 4.0 International License, which permits use, sharing, adaptation, distribution and reproduction in any medium or format, as long as you give appropriate credit to the original author(s) and the source, provide a link to the Creative Commons licence, and indicate if changes were made. The images or other third party material in this article are included in the article's Creative Commons licence, unless indicated otherwise in a credit line to the material. If material is not included in the article's Creative Commons licence and your intended use is not permitted by statutory regulation or exceeds the permitted use, you will need to obtain permission directly from the copyright holder. To view a copy of this licence, visit http://creativecommons.org/licenses/ by $/ 4.0 /$.

\section{References}

1. Dimitriadis, G.: Introduction to Nonlinear Aeroelasticity. Wiley, Hoboken (2017)

2. Bisplinghoff, R.L., Ashley, H., Halfman, R.L.: Aeroelasticity. Courier Corporation, North Chelmsford (2013)

3. Dowell, E.H.: A Modern Course in Aeroelasticity. Springer, Berlin (2015)

4. Fung, Y.C.: An Introduction to the Theory of Aeroelasticity. Courier Dover Publications, Mineola (2008)

5. Balakrishnan, A.V.: Aeroelasticity: The Continuum Theory. Springer, Berlin (2012)

6. Hodges, D.H., Pierce, G.A.: Introduction to Structural Dynamics and Aeroelasticity, vol. 15. Cambridge University Press, Cambridge (2011)

7. Lee, B., Price, S., Wong, Y.: Nonlinear aeroelastic analysis of airfoils: bifurcation and chaos. Prog. Aerosp. Sci. 35(3), 205-334 (1999)

8. Dowell, E., Edwards, J., Strganac, T.: Nonlinear aeroelasticity. J. Aircr. 40(5), 857-874 (2003)
9. O’Neil, T., Strganac, T.W.: Aeroelastic response of a rigid wing supported by nonlinear springs. J. Aircr. 35(4), 616622 (1998)

10. Price, S., Alighanbari, H., Lee, B.: The aeroelastic response of a two-dimensional airfoil with bilinear and cubic structural nonlinearities. J. Fluids Struct. 9(2), 175-193 (1995)

11. Dowell, E., Ueda, T.: Flutter analysis using nonlinear aerodynamic forces. J. Aircr. 21(2), 101-109 (1984)

12. Tang, D., Dowell, E.: Comparison of theory and experiment for non-linear flutter and stall response of a helicopter blade. J. Sound Vib. 165(2), 251-276 (1993)

13. Wang, L., Liu, X., Kolios, A.: State of the art in the aeroelasticity of wind turbine blades: aeroelastic modelling. Renew. Sustain. Energy Rev. 64, 195-210 (2016)

14. Farhat, C.: CFD-based nonlinear computational aeroelasticity. In: Stein, E., Borst, R., Hughes, T.J.R. (eds.) Encyclopedia of Computational Mechanics, 2nd edn, pp. 1-21. Wiley, Hoboken (2017)

15. Bombardieri, R., Cavallaro, R., de Teresa, J. L. Sáez, Karpel, M.: Nonlinear aeroelasticity: a CFD-based adaptive methodology for flutter prediction. In: AIAA Scitech 2019 Forum, p. 1866 (2019)

16. Gilliatt, H.C., Strganac, T.W., Kurdila, A.J.: An investigation of internal resonance in aeroelastic systems. Nonlinear Dyn. 31(1), 1-22 (2003)

17. Dimitriadis, G., Li, J.: Bifurcation behavior of airfoil undergoing stall flutter oscillations in low-speed wind tunnel. AIAA J. 47(11), 2577-2596 (2009)

18. dos Santos, C.R., Pereira, D.A., Marques, F.D.: On limit cycle oscillations of typical aeroelastic section with different preset angles of incidence at low airspeeds. J. Fluids Struct. 74, 19-34 (2017)

19. Jian, Z., Jinwu, X.: Nonlinear aeroelastic response of highaspect-ratio flexible wings. Chin. J. Aeronaut. 22(4), 355363 (2009)

20. Sarkar, S., Bijl, H.: Nonlinear aeroelastic behavior of an oscillating airfoil during stall-induced vibration. J. Fluids Struct. 24(6), 757-777 (2008)

21. Bose, C., Gupta, S., Sarkar, S.: Transition to chaos in the flow-induced vibration of a pitching-plunging airfoil at low Reynolds numbers: Ruelle-Takens-Newhouse scenario. Int. J. Non-Linear Mech. 109, 189-203 (2019)

22. Kalmár-Nagy, T., Csikja, R., Elgohary, T.A.: Nonlinear analysis of a 2-DOF piecewise linear aeroelastic system. Nonlinear Dyn. 85(2), 739-750 (2016)

23. Liu, L., Wong, Y., Lee, B.: Non-linear aeroelastic analysis using the point transformation method, part 1: freeplay model. J. Sound Vib. 253(2), 447-469 (2002)

24. Liu, L., Wong, Y., Lee, B.: Non-linear aeroelastic analysis using the point transformation method, part 2: hysteresis model. J. Sound Vib. 253(2), 471-483 (2002)

25. Chung, K., Chan, C., Lee, B.: Bifurcation analysis of a twodegree-of-freedom aeroelastic system with freeplay structural nonlinearity by a perturbation-incremental method. J. Sound Vib. 299(3), 520-539 (2007)

26. Trickey, S., Virgin, L., Dowell, E.: Characterizing stability of responses in a nonlinear aeroelastic system. In: 41st Structures, Structural Dynamics, and Materials Conference and Exhibit, p. 1334 (2000)

27. Sales, T d P, Pereira, D.A., Marques, F.D., Rade, D.A.: Modeling and dynamic characterization of nonlinear non-smooth 
aeroviscoelastic systems. Mech. Syst. Signal Process. 116, 900-915 (2019)

28. Radcliffe, T., Cesnik, C.: Aeroelastic response of multisegmented hinged wings. In: 19th AIAA Applied Aerodynamics Conference, p. 1371 (2001)

29. Dimitriadis, G., Vio, G., Cooper, J.: Application of higher-order harmonic balance to non-linear aeroelastic systems. In: 47th AIAA/ASME/ASCE/AHS/ASC Structures, Structural Dynamics, and Materials Conference 14th AIAA/ASME/AHS Adaptive Structures Conference 7th, p. 2023 (2006)

30. Jones, D., Roberts, I., Gaitonde, A.: Identification of limit cycles for piecewise nonlinear aeroelastic systems. J. Fluids Struct. 23(7), 1012-1028 (2007)

31. Liao, H.: Piecewise constrained optimization harmonic balance method for predicting the limit cycle oscillations of an airfoil with various nonlinear structures. J. Fluids Struct. 55, 324-346 (2015)

32. Sun, Z., Haghighat, S., Liu, H.H., Bai, J.: Time-domain modeling and control of a wing-section stall flutter. J. Sound Vib. 340, 221-238 (2015)

33. Goodman, C.: Accurate subcritical damping solution of flutter equation using piecewise aerodynamic function. J. Aircr. 38(4), 755-763 (2001)

34. Andronov, A.A., Vitt, A.A., Khaikin, S.E.: Theory of Oscillators. Pergamon Press, Oxford (1966)

35. Magri, L., Galvanetto, U.: Example of a non-smooth Hopf bifurcation in an aero-elastic system. Mech. Res. Commun. 40, 26-33 (2012)

36. Frahm, H.: Device for damping vibrations of bodies. April 18. US Patent 989,958 (1911)

37. Den Hartog, J.P.: Mechanical Vibrations. Courier Corporation, North Chelmsford (1985)

38. Gattulli, V., Di Fabio, F., Luongo, A.: Nonlinear tuned mass damper for self-excited oscillations. Wind Struct. 7(4), 251264 (2004)

39. Malher, A., Touzé, C., Doaré, O., Habib, G., Kerschen, G.: Flutter control of a two-degrees-of-freedom airfoil using a nonlinear tuned vibration absorber. J. Comput. Nonlinear Dyn. 12(5), 051016 (2017)

40. Kassem, M., Yang, Z., Gu, Y., Wang, W., Safwat, E.: Active dynamic vibration absorber for flutter suppression. J. Sound Vib. 469, $115110(2020)$

41. Gilliatt, H., Strganac, T., Kurdila, A., Gilliatt, H., Strganac, T., Kurdila, A.: Nonlinear aeroelastic response of an airfoil. In: 35th Aerospace Sciences Meeting and Exhibit, p. 459 (1997)

42. Sheldahl, R.E., Klimas, P.C.: Aerodynamic characteristics of seven symmetrical airfoil sections through 180-degree angle of attack for use in aerodynamic analysis of vertical axis wind turbines. Technical report, Sandia National Laboratories. SAND80-2114 (1981)

43. Abbott, I., Von Doenhoff, A., Stivers, L.: NACA report no. 824-summary of airfoil data. National Advisory Committee for Aeronautics (1945)
44. Barenblatt, G.I., Barenblatt, G.I., Isaakovich, B.G.: Scaling, Self-Similarity, and Intermediate Asymptotics: Dimensional Analysis and Intermediate Asymptotics, vol. 14. Cambridge University Press, Cambridge (1996)

45. Di Bernardo, M., Budd, C., Champneys, A.R., Kowalczyk, P.: Piecewise-Smooth Dynamical Systems: Theory and Applications, vol. 163. Springer, Berlin (2008)

46. Di Bernardo, M., Pagano, D.J., Ponce, E.: Nonhyperbolic boundary equilibrium bifurcations in planar Filippov systems: a case study approach. Int. J. Bifurc. Chaos 18(5), 1377-1392 (2008)

47. Gantmacher, F.R.: The Theory of Matrices, vol. 2. Chelsea, New York (1959)

48. Di Bernardo, M., Nordmark, A., Olivar, G.: Discontinuityinduced bifurcations of equilibria in piecewise-smooth and impacting dynamical systems. Physica D 237(1), 119-136 (2008)

49. Simpson, D.J.W.: Bifurcations in Piecewise-Smooth Continuous Systems, vol. 70. World Scientific, Singapore (2010)

50. Freire, E., Ponce, E., Rodrigo, F., Torres, F.: Bifurcation sets of continuous piecewise linear systems with two zones. Int. J. Bifurc. Chaos 8(11), 2073-2097 (1998)

51. Simpson, D.J., Kuske, R.: Mixed-mode oscillations in a stochastic, piecewise-linear system. Physica D 240(14-15), 1189-1198 (2011)

52. Ponce, E., Ros, J., Vela, E.: The focus-center-limit cycle bifurcation in discontinuous planar piecewise linear systems without sliding. In: Ibáñez, S., Pérez del Río, J.S., Pumariño, A., Rodríguez, J.Á. (eds.) Progress and Challenges in Dynamical Systems, pp. 335-349. Springer, Berlin (2013)

53. Kriegsmann, G.: The rapid bifurcation of the Wien bridge oscillator. IEEE Trans. Circuits Syst. 34(9), 1093-1096 (1987)

54. Li, N., Balas, M.J., Nikoueeyan, P., Yang, H., Naughton, J.W.: Stall flutter control of a smart blade section undergoing asymmetric limit oscillations. Shock Vib. 2016, 1-14 (2016)

55. Razak, N.A., Andrianne, T., Dimitriadis, G.: Flutter and stall flutter of a rectangular wing in a wind tunnel. AIAA J. 49(10), 2258-2271 (2011)

56. Dunn, P., Dugundji, J.: Nonlinear stall flutter and divergence analysis of cantilevered graphite/epoxy wings. AIAA J. 30(1), 153-162 (1992)

Publisher's Note Springer Nature remains neutral with regard to jurisdictional claims in published maps and institutional affiliations. 